\title{
Computational Aerodynamics of Low Reynolds Number Plunging, Pitching and Flexible Wings for MAV Applications
}

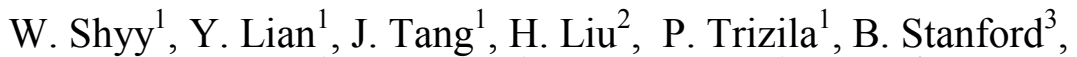 \\ L. Bernal ${ }^{1}$, C. Cesnik ${ }^{1}$, P. Friedmann ${ }^{1}$, P. Ifju ${ }^{3}$ \\ ${ }^{1}$ Department of Aerospace Engineering \\ University of Michigan \\ Ann Arbor, MI 48109, U.S.A. \\ ${ }^{2}$ Graduate School of Engineering \\ Chiba University \\ Chiba, 263-8522, Japan \\ ${ }^{3}$ Department of Mechanical and Aerospace Engineering \\ University of Florida \\ Gainesville, FL 32611-6250, U.S.A
}

\begin{abstract}
Micro Air Vehicles (MAVs) have the potential to revolutionize our sensing and information gathering capabilities in environmental monitoring and homeland security areas. Due to the MAVs' small size, flight regime, and modes of operation, significant scientific advancement will be needed to create this revolutionary capability. Aerodynamics, structural dynamics, and flight dynamics of natural flyers intersects with some of the richest problems in MAVs, including massively unsteady three-dimensional separation, transition in boundary layers and shear layers, vortical flows and bluff body flows, unsteady flight environment, aeroelasticity, and nonlinear and adaptive control are just a few examples. A challenge is that the scaling of both fluid dynamics and structural dynamics between smaller natural flyer and practical flying hardware/lab experiment (larger dimension) is fundamentally difficult. In this paper, we offer an overview of the challenges and issues, along with sample results illustrating some of the efforts made from a computational modeling angle.
\end{abstract}

\section{INTRODUCTION}

Micro Air Vehicles (MAVs) have the potential to revolutionize our capabilities of gathering information in environmental monitoring, homeland security, and other time sensitive areas. To meet the evolving threat, MAVs must have the ability to fly in urban settings, tunnels and caves, maintain forward and hovering flight, maneuver in constrained environments, and "perch" until needed. Due to the MAVs' small size, flight regime, and modes of operation, significant scientific advancement will be needed to create this revolutionary capability. Insufficient knowledge, predictive capabilities, and experimental data exist regarding the fundamental unsteady aerodynamics of low Reynolds number flyers, and the associated fluid-structure-control interactions, flight mechanics, guidance and control. From a biology-inspired viewpoint, aerodynamics, structural dynamics, and flight dynamics of birds, bats, and insects intersects with some of the richest problems in aerospace engineering: massively unsteady three-dimensional separation, transition in boundary layers and shear layers, vortical flows and bluff body 
flows, unsteady flight environment, aeroelasticity, and nonlinear and adaptive control are just a few examples. The large flexibility of animal wings leads to complex fluidstructure interactions, while the kinematics of flapping and the often spectacular maneuvers performed by natural flyers result in highly coupled nonlinearities in fluid mechanics, aeroelasticity, flight dynamics, and control systems. The agility and flight performance of natural flyers is of particular interest to the aerospace community, from the viewpoints of both fundamental engineering science and the development of miniaturized flight vehicles. For all of the maturity of aerodynamics as an engineering discipline, our understanding of flight in natural flyers presently stands far from complete.

There are several distinct features of natural flyers in their flight characteristics. For example, (i) for natural flyers, there is substantial anisotropy in the structural characteristics between the chordwise and spanwise directions, (ii) natural flyers employ shape control to accommodate spatial and temporal flow structures, (iii) natural flyers accommodate wind gust and accomplish station keeping with several established kinematics patterns, (iv) natural flyers utilize multiple unsteady aerodynamic mechanisms for lift and thrust enhancement, and (v) natural flyers combine sensing, control and wing maneuvering to maintain not only lift but also flight stability. In principle, one might like to first understand a biological system, then abstract certain properties and apply them to MAV design. A challenge is that the scaling of both fluid dynamics and structural dynamics between smaller natural flyer and practical flying hardware/lab experiment (larger dimension) is fundamentally difficult. Regardless, in order to develop a satisfactory flyer, one needs to meet the following objectives:

- generate necessary lift, which scales with the vehicle/wing length scale as $\ell^{3}$ (under geometric similitude); however, oftentimes, a flyer needs to increase or reduce lift to maneuver toward/avoid an object, resulting in substantially more complicated considerations;

- minimize the power consumption.

An optimal design based on a single design point, under a given steady freestream value, is insufficient; instead, we need to develop knowledge base guiding future design of MAVs across a range of wind gust and flight speed and time scales so that they can be optimal flyers defined by the entire flight envelop.

In this paper, we will offer our perspective regarding the issues, progress, and challenges associated with unsteady low Reynolds number aerodynamics pertaining to MAV development. In particular, we will discuss plunging, pitching and flexible wings. In the following, we will first review the scaling parameters, and then discuss kinematics used by natural flyers. The wing flexibility and its implications will also be reviewed.

\section{SCALING, FLAPPING KINEMATICS \& WING STRUCTURES}

\section{(i) Parameter Space and Scaling Laws}

From the viewpoint of fluid and structural dynamics, there are different dimensionless parameters that are of relevance to our study. Consider: $c$, chord length; $f$, flapping 
frequency; $h_{a}$, flapping amplitude; $U_{r e f}$, reference velocity; $v$ kinematic viscosity; $\rho_{f}$, fluid density; $D$, plate stiffness (directly proportional to material Young's modulus and the cube of the wing thickness); $I_{B}$, (flapping) moment of inertia; $f_{n}$, wing natural frequencies. The relevant dimensionless parameters are listed in Table 1. Assuming that the geometric similarity is maintained, the scaling laws for forward and hovering flight conditions are summarized in the same table. One can readily conclude that the hovering Reynolds number and the cruising Reynolds number are very close to each other because the characteristic velocity for hovering is $U_{r e f}=2 \pi f h_{a}$. For hovering, the reduced frequency becomes $k=c / 2 h_{a}$, which is simply related to the normalized stroke amplitude. Furthermore, if we use the forward flight speed as the velocity scale, then the resulting non-dimensional form of the momentum equation explicitly contains the Reynolds number and the Strouhal number. On the other hand, if we choose to use the flapping velocity scale, then the momentum equation will explicitly contain the Reynolds number and the reduced frequency [1]. As shown on Table 1, the scaling laws make the construction of aeroelastic models and testing complicated. Moreover, it leads to the usage of structural materials with elastic properties that are different from those of the structure of the natural flyer.

Table 1. Dimensionless parameters and scaling dependency for flapping wings

\begin{tabular}{|l|l|l|l|l|}
\hline \multirow{2}{*}{$\begin{array}{l}\text { Dimensionless } \\
\text { Parameter }\end{array}$} & $\begin{array}{l}\text { Hovering } \\
\text { Based on flapping wing speed }\end{array}$ & \multicolumn{2}{l|}{$\begin{array}{l}\text { Forward Flight } \\
\text { Based on cruising speed }\end{array}$} \\
\cline { 2 - 5 } & length & frequency & length & frequency \\
\hline $\begin{array}{l}\text { Reynolds number: } \\
R_{e}=U_{\text {ref }} c / v\end{array}$ & $\ell^{2}$ & $f$ & $\ell$ & independent \\
\hline $\begin{array}{l}{ }^{1} \text { Strouhal number: } \\
S t=\left(2 f h_{a}\right) / U_{\text {ref }}\end{array}$ & independent & independent & $\ell$ & $f$ \\
\hline $\begin{array}{l}\text { Reduced } \\
\text { frequency: } \\
\mathrm{k}=\pi f c / U_{\text {ref }}\end{array}$ & independent & independent & $\ell$ & $f$ \\
\hline${ }^{2} \Pi_{1}=\frac{D}{\rho_{f} U_{r e f}^{2} c^{3}}$ & $\ell^{-2}$ & $f^{-2}$ & independent & independent \\
\hline${ }^{3} \Pi_{2}=\frac{I_{B}}{\rho_{f} c^{5}}$ & $\ell^{-1}$ & independent & $\ell^{-1}$ & independent \\
\hline $\begin{array}{l}\text { Frequency ratios: } \\
\Pi_{3}=f_{n} / f\end{array}$ & $\ell^{-1}$ & $f^{-1}$ & $\ell^{-1}$ & $f^{-1}$ \\
\hline
\end{tabular}

Table 2 summarizes some key parameters of three natural flyers. The three natural flyers are: bumblebee, hawkmoth, and hummingbird. The chord Reynolds number is $\mathrm{O}\left(10^{3}\right)$ for

\footnotetext{
${ }^{1}$ It is noted that the advance ratio, $J=U_{r e f}\left(2 \pi f h_{a}\right)$ is related to $S t$, specifically, $J=1 /(S t . \pi)$.

${ }^{2}$ Ratio of elastic and aerodynamic forces. $\Pi_{1}$ gives a relative measure of elastic deformation to given aerodynamic loading and it is important as a measure of the structural nonlinear regime. $\Pi_{1}$ is also related to the Kussner factor for flutter estimation. It is uncertain, however, the applicability of the latter to the flapping wing stability boundary, and this should be investigated.

${ }^{3}$ Ratio of inertia and aerodynamic generalized forces. $\Pi_{2}$ is related to the Lock number and it contains the mass ratio (representing the relative density of the wing and the fluid surrounding it).
} 
bees, and $\mathrm{O}\left(10^{4}\right)$ for moths and hummingbirds. In such an intermediate Reynolds number both inertial and viscous forces are equivalently important, generally resulting in highly unsteady, complicated vortical flows. The interaction and integration between aerodynamics, structural flexibility, and control strategies are a function of the flow regime and their complexity has been the limiting factor for in depth understanding of MAV flapping wing performance, response and stability.

Table 2. Morphological and flight parameters of selected natural flyers

\begin{tabular}{|c|c|c|c|c|c|}
\hline Parameters & & & $\begin{array}{l}\text { Bumblebee } \\
\text { (Bombus terrestris) }\end{array}$ & $\begin{array}{l}\text { Hawkmoth } \\
\text { (Manduca sexta) }\end{array}$ & $\begin{array}{l}\text { Hummingbird } \\
\text { (Lampornis } \\
\text { clemenciae) }\end{array}$ \\
\hline \multicolumn{6}{|c|}{ Morphological parameters } \\
\hline Total mass (body mass) & & {$[\mathrm{mg}]$} & 170 & 1600 & 8400 \\
\hline Wing mass & $\mathbf{m}_{\mathbf{w}}$ & {$[\mathrm{mg}]$} & 0.9 (both wings) & 90 (both wings) & 600 (both wings) \\
\hline Wing length & $\mathbf{R}$ & {$[\mathrm{mm}]$} & 13.2 & 48.5 & 85 \\
\hline Wing area & $\mathbf{S}$ & {$\left[\mathrm{mm}^{2}\right]$} & 100 (both wings) & 1800 (both wings) & 3500 (both wings) \\
\hline \multicolumn{6}{|l|}{ Flight parameters } \\
\hline Flapping frequency & f & {$[\mathrm{Hz}]$} & 150 & 25 & 25 \\
\hline Stroke amplitude & $\Phi$ & [rad] & 2 & 2 & 2.5 \\
\hline Chord Reynolds number & & $\operatorname{Re}$ & $1200-3000$ & 5000 & $10,000-15,000$ \\
\hline
\end{tabular}

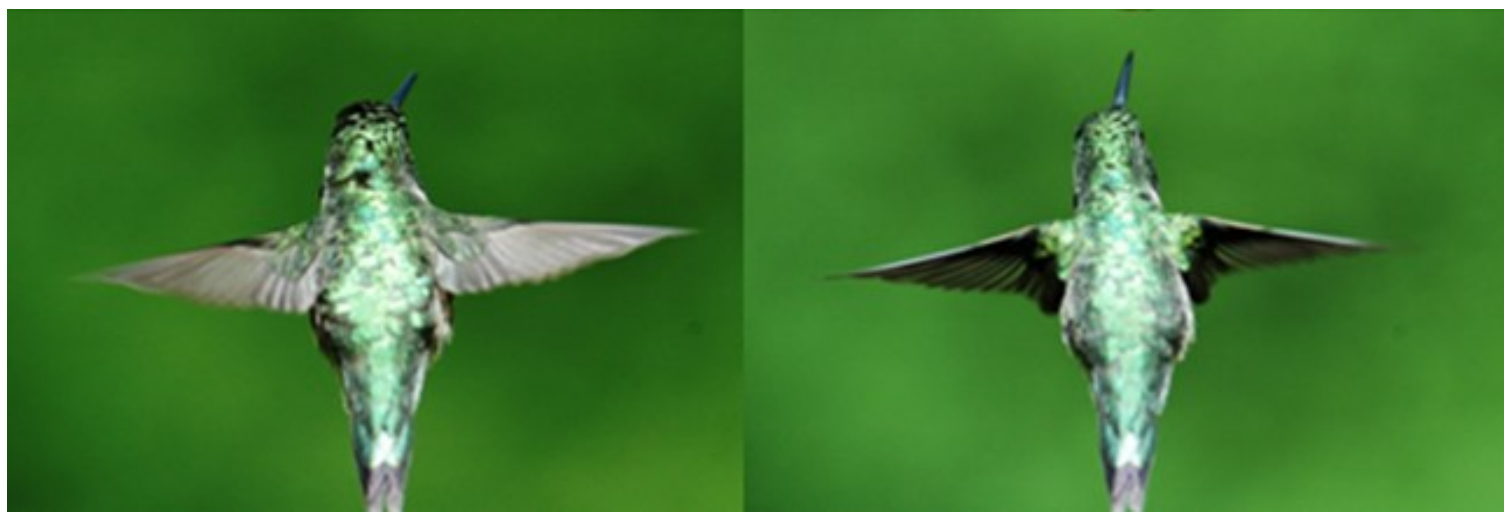

Figure 1._Symmetric, figure-eight flapping pattern of a hummingbird.

\section{(ii) Flapping Kinematics \& Wing Morphology}

Flapping kinematics can be very complicated. For example, the figure-eight pattern can be found in flies, hummingbirds and other small flyers (Figure 1). (AoA in fruit fly wing varies more spanwise) Furthermore, the AoA along the spanwise direction can show substantial variation. Figure 2 shows snapshots of a robin and a red-wing blackbird with their wings fully extended. Of course, the wing motion is complicated and instantaneous wing shapes cannot capture the essence of the entire aerodynamic implications. Nevertheless, these images do offer us insight into aerodynamic model development.

When wind gust adjustment, object avoidance, or station keeping become major factors, highly deformed wing shapes, and coordinated wing-tail movement become clear. Figure 3 illustrates such behavior for a hummingbird maneuvering around a potential threat, a 
chickadee adjusting its flight path to accommodate a target, and a finch making a precision landing. Figure 4 shows several flight modes of a cardinal. Understanding of the aerodynamic, structural and control implications of these modes is essential for the development of high performance, robust micro air vehicles capable of performing desirable missions.

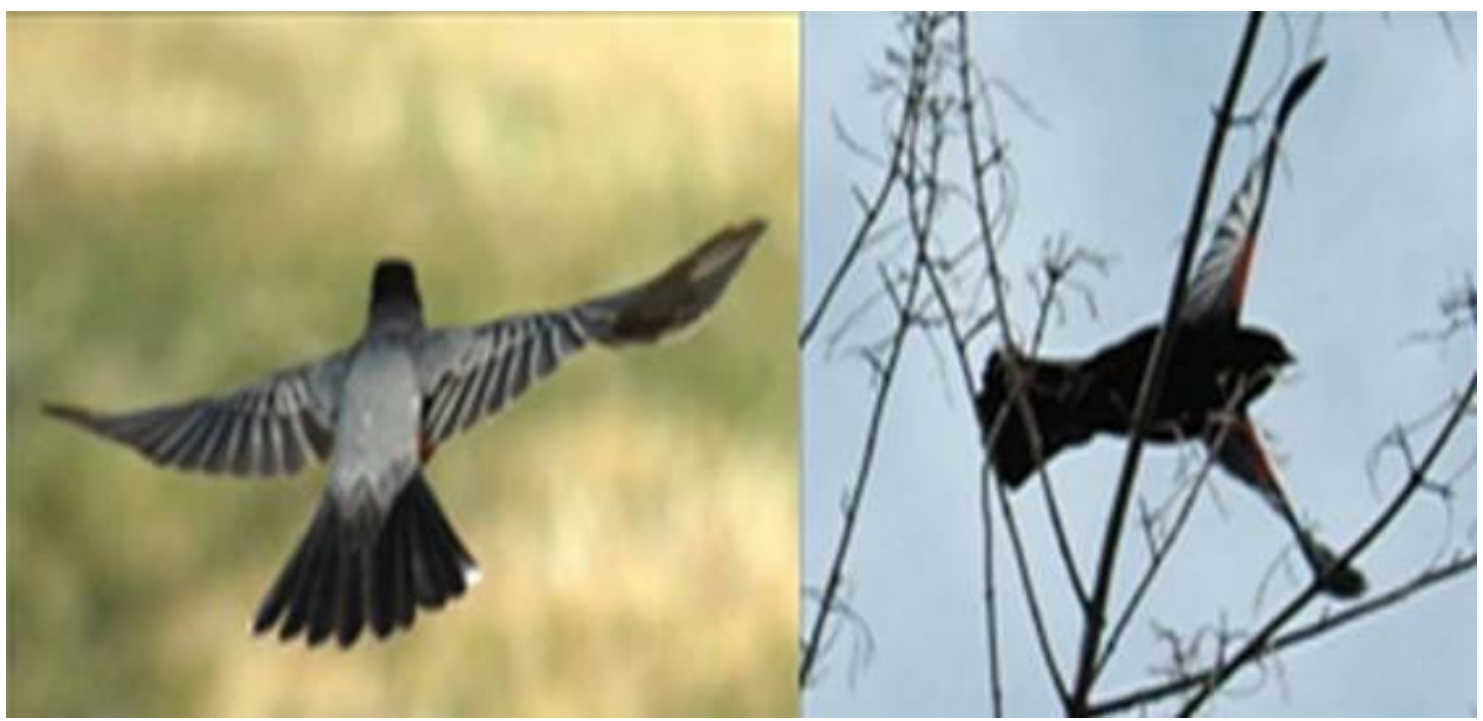

Figure 2. Variable AoA along the spanwise direction; left- robin, right- redwing blackbird.

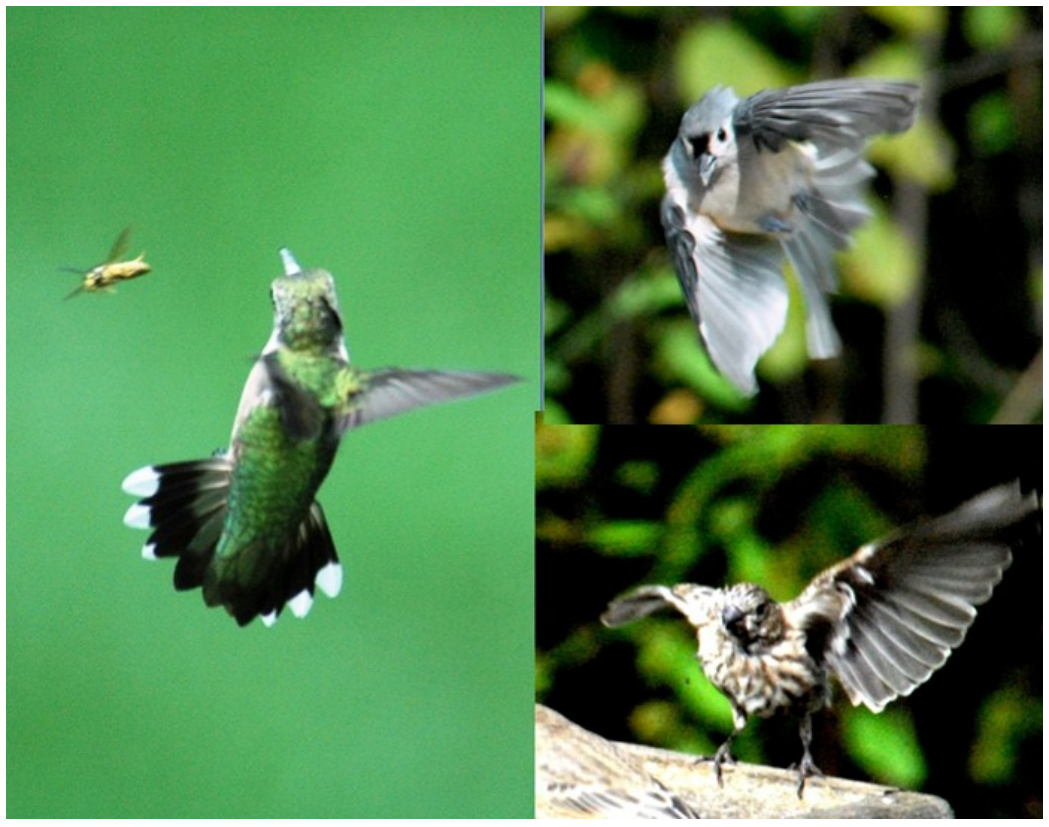

Figure 3. Asymmetric flapping kinematics, involving wing-tail coordination, are displayed with a hummingbird avoiding a potential threat, a nuthatch making adjustment while flying toward a target, and a finch during landing. 


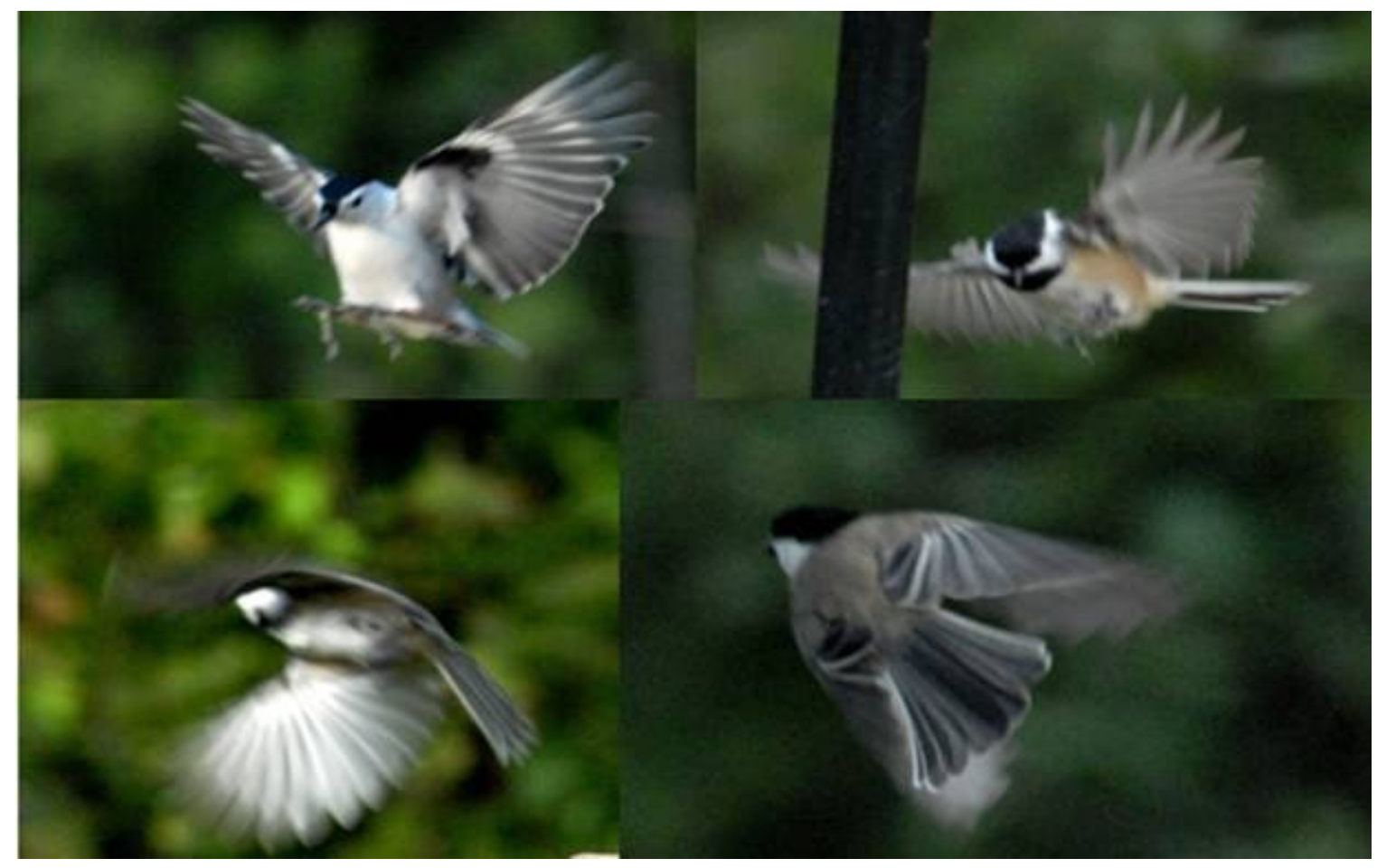

Figure 4. Selected flight modes of a chickadee, including fully spread wings, downward flapping, asymmetric wing-tail combinations, and partially folded wings during descend.

\section{(iii) Flexible Wing Structures}

Insect wings display anisotropic properties because of the membrane-batten structures. Figure 5 shows wings of dragonfly, cicada and wasp. They exhibit substantial variations in aspect ratios and shapes but share a common feature of reinforced leading edge. A dragonfly wing has more local variations in its structural composition, and is more corrugated than a cicada or a wasp wing. In a fixed wing set-up, wind tunnel measurements show that such corrugated wings seem aerodynamically insensitive to the Reynolds number variations, which is quite different from a typical low Reynolds number airfoil. For example, Figure 6 shows that a dragonfly wing is insensitivity to the Reynolds number in its operating range, in contrast to the Eppler E374 airfoil, which displays a zigzag pattern in certain Reynolds number range. 


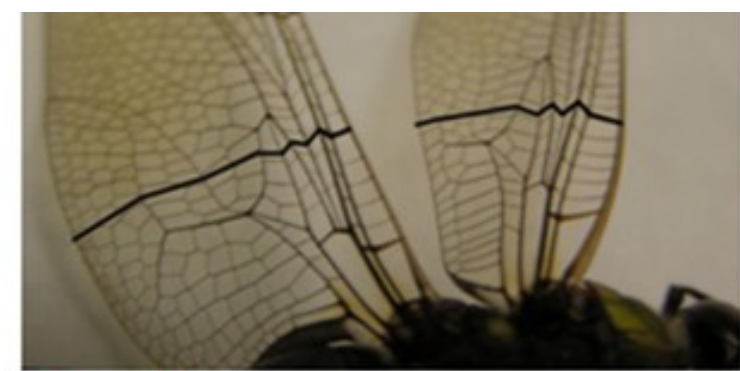

Dragonfly wings.

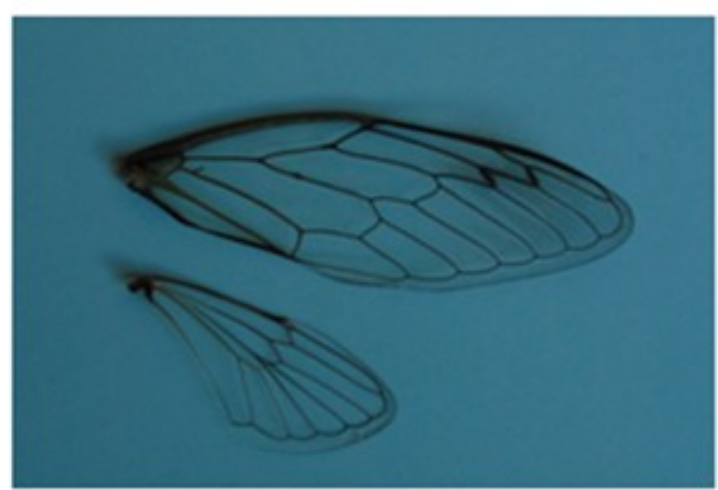

Cicada wings: less corrugated, less peripherally reinforced than dragonfly wing

Figure 5. Wing structures of dragonfly, cicada and wasp, with reinforced leading edge anisotropic mechanical property distributions, and corrugated geometries.
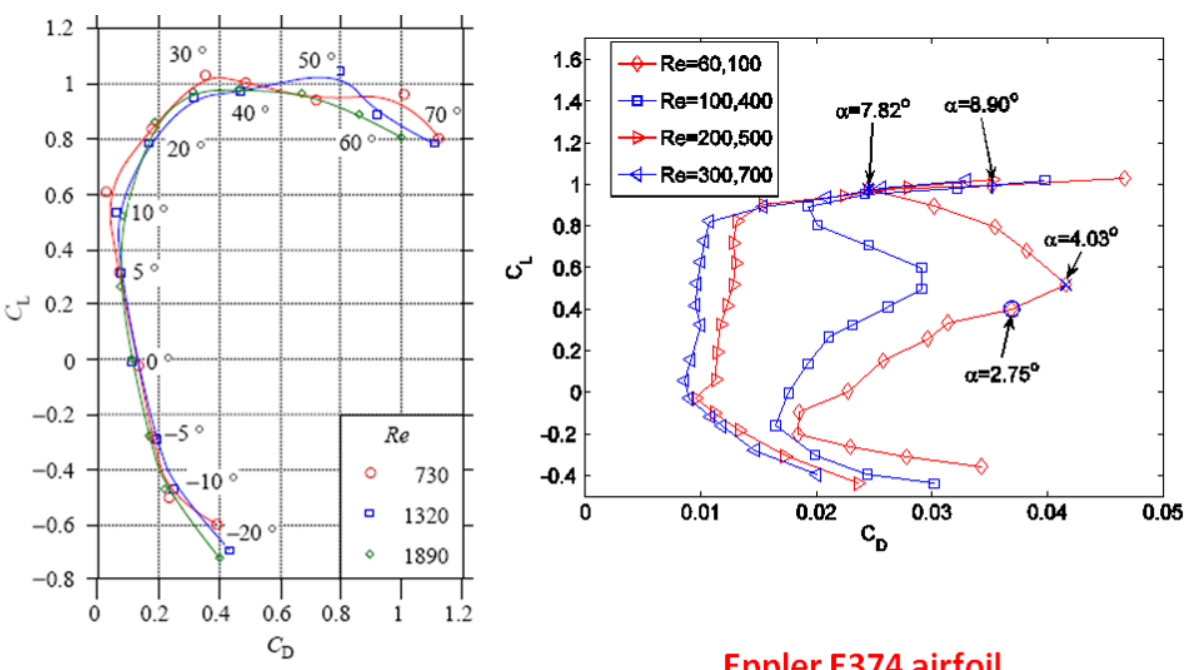

Eppler E374 airfoil

Dragonfly forewing

Figure 6. Lift-drag polars of dragonfly forewing [2] and Eppler E374 airfoil [3].

The membrane-like flexible wing structures are observed in insects, bats, and birds. Figure 7 shows wings of hummingbird, bat, wasp and bumblebee, all exhibiting these similar overall structural characteristics. 

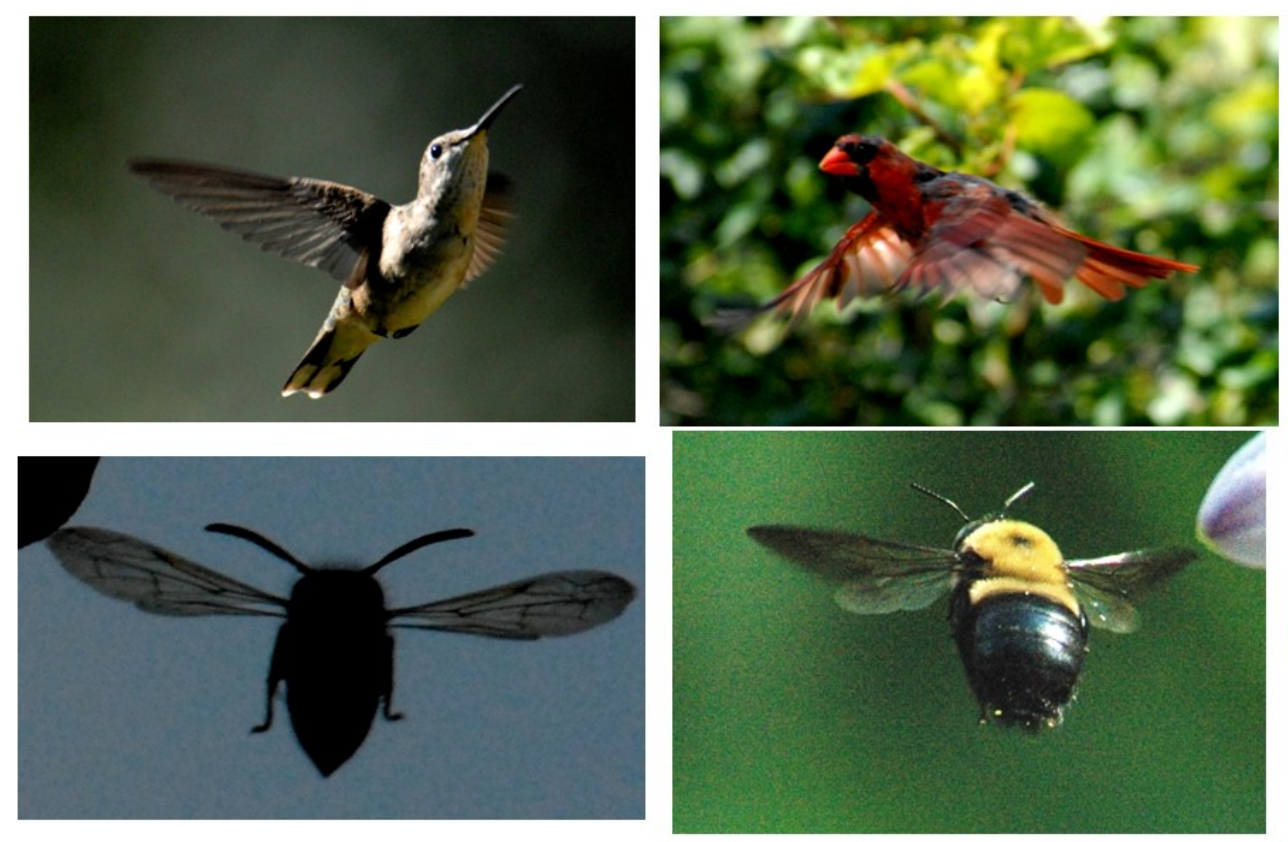

Figure 7. Wings of hummingbird, bat, wasp and bumblebee all show similar battenmembrane appearances when flapping.

\section{RECENT INVESTIGATIONS OF AERODYNAMICS}

\section{(i) Leading Edge Vortex (LEV)}

The LEV is a common feature associated with low Re flapping wing aerodynamics; the flow structures are influenced by the swirl strength, the Reynolds number, as well as the rotational rates. Its effectiveness in promoting lift is correlated with a flyer's size. As reviewed in [1, 4] and highlighted in [5, 6], in addition to the LEV, numerous issues related to the interplay among wing structures (including its anisotropic deformability), flapping kinematics, large vortex structures and Reynolds number remain unresolved. They are critical for advancing concepts and technologies for future MAVs, and should be investigated thoroughly.

Figure 8 illustrates the evolution of LEV during one flapping cycle. The airfoil starts from the upper top [7]. As it descends, a small LEV emerges near the lead edge. The LEV grows in size and strength and travels downstream as the airfoil continues to move downward. When the airfoil moves close to its lowest position, the LEV breaks into two vortices. The two vortices shed into the wake and gradually lose their strength and coherent structure. Figure 9 shows the pressure distributions at different time instants. Referring to the vorticity contours in Figure 8 we can see that the LEV creates a low pressure zone on the airfoil surface. This low pressure zone enhances both the lift and thrust. 


$$
h(t)=h_{a} \cos \left(2 \pi f t+\phi_{h}\right)
$$

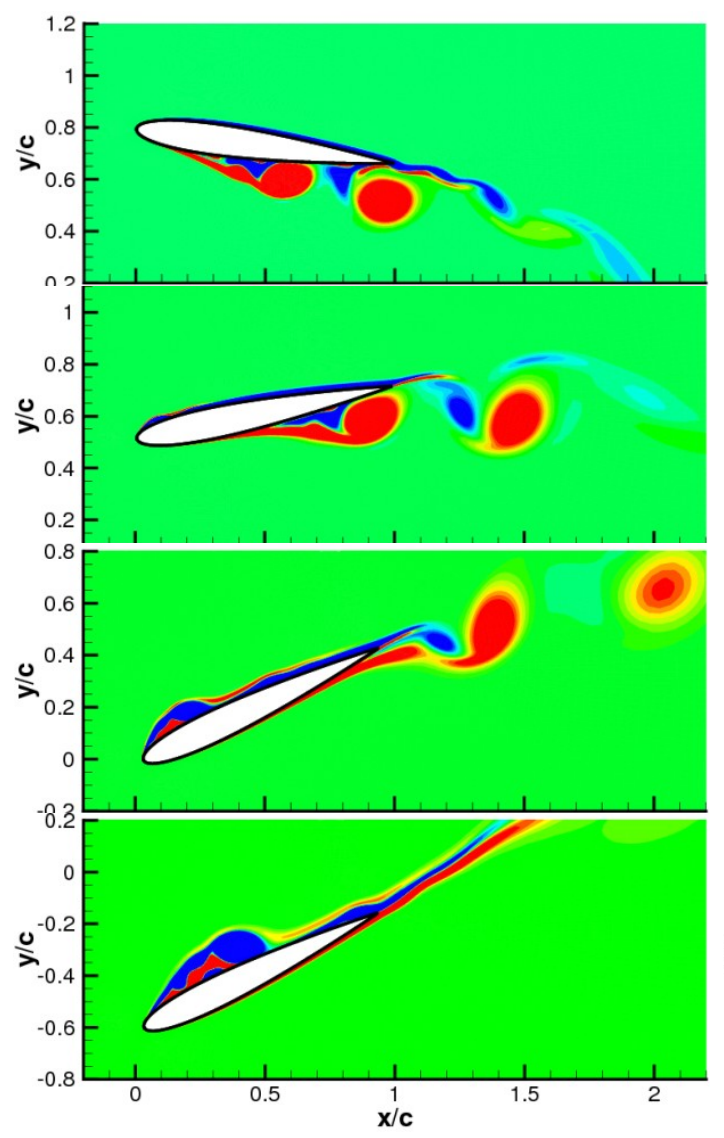

$$
\alpha(t)=\alpha_{0}+\alpha_{a} \cos \left(\omega t+\phi_{\theta}\right)
$$

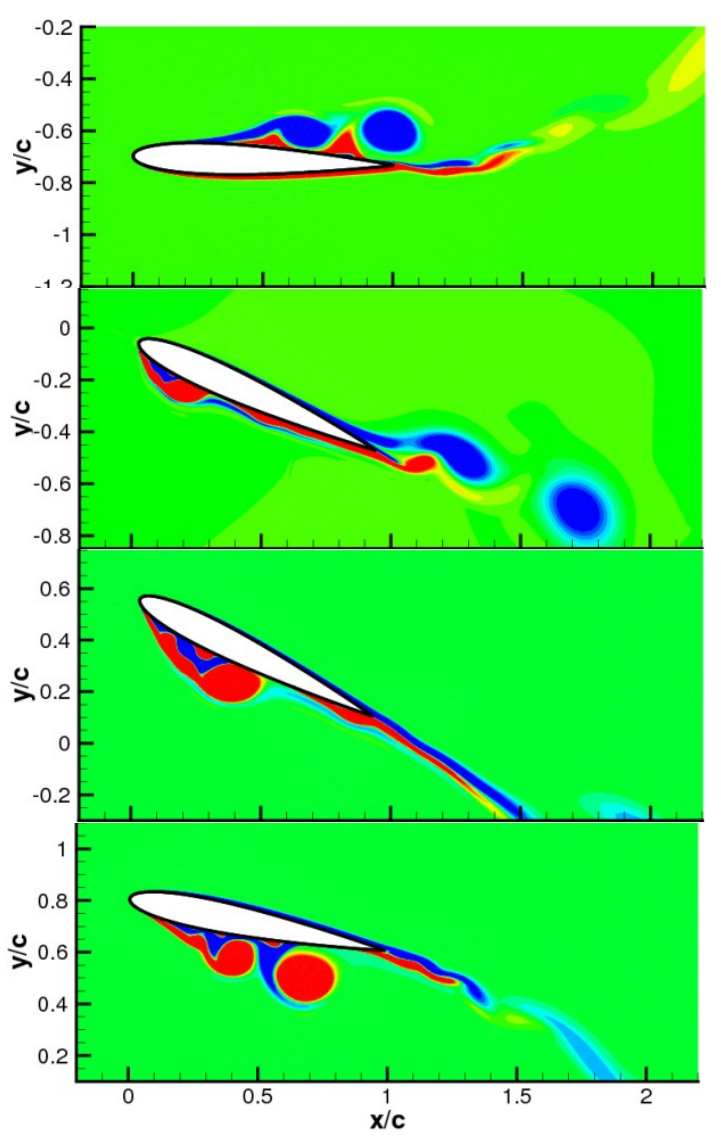

Figure 8. Vorticity contours at different instants during one flapping cycle. $\mathrm{Re}=4 \times 10^{4}, h_{\mathrm{a}}$ $=0.75 \mathrm{c}, S t=0.3, k=0.63, \varphi=\varphi_{\theta^{-}} \varphi_{h}=75^{\circ}, \alpha_{0}=0$, and $\alpha_{0}=28^{\circ}$.

For three-dimensional cases, the situation becomes complex. At this time, there is a controversy concerning the role of the LEV in enhancing aerodynamic lift during flapping flight. Ellington et al. [8] investigated the aerodynamics of hawkmoths, and first suggested that the LEV can significantly promote lift associated with a flapping wing. There have been multiple follow-up investigations [e.g., 9-13] based on different insect models, resulting in varied views on the role played by the LEV and implications on lift generation. For $\mathrm{Re}$ around $\mathrm{O}\left(10^{3}\right.$ to $\left.10^{4}\right)$, corresponding to larger insects such as hawkmoths, the LEV can enhance lift by attaching a bounded vortex core to the upper leading edge during wing translation. To be effective in enhancing lift, the LEV needs to maintain a high axial flow velocity in the core and remains stable along the spanwise direction, before separating from the wing at, say, $75 \%$ of the spanwise location toward the wing tip, and then connecting to a tip vortex. The overall vortical structures are qualitatively similar to those of low aspect-ratio delta wings $[1,9]$ which stabilize the LEV due to the spanwise pressure-gradient, increasing lift well above the critical angle of attack. In essence, the vortex stability in flapping wings is maintained by a spanwise axial flow along the vortex core, creating 'delayed stall', to enhance lift during the translational phase. 


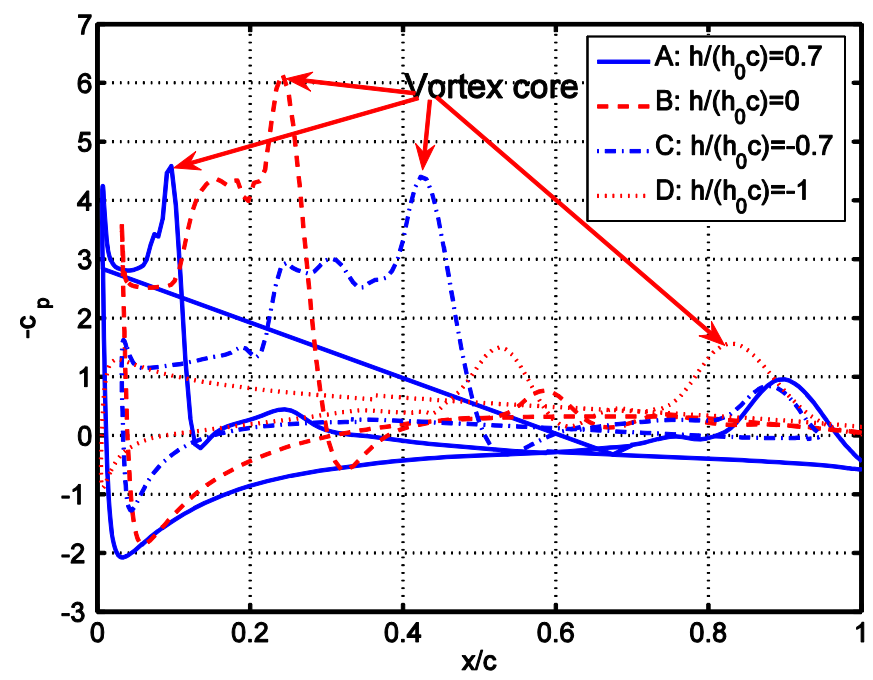

Figure 9. Pressure coefficient distributions at three time instants. $\mathrm{Re}=4 \times 10^{4}, h_{\mathrm{a}}=0.75 \mathrm{c}, S t$ $=0.3, k=0.63, \varphi=\varphi_{\theta}-\varphi_{h}=75^{\circ}, \alpha_{0}=0$, and $\alpha_{0}=28^{\circ}$.

Birch and Dickinson [12] investigated the LEV related to the fruit fly at the Reynolds number of 160. They report that, in contrast to the hawkmoth LEV, the LEV of fruit fly exhibits a stable vortex structure without separation during most of the translational phases. Furthermore, there is little axial flow in the vortex core, amounting to only 2-5\% of the averaged tip velocity. Observing the considerable difference exhibited between fruit fly and hawkmoth models, Birch \& Dickinson [12] hypothesized that the attenuating effect of the downwash induced by the tip vortex and wake vorticity, limits the growth of the LEV by lowering the effective angle of attack and prolongs the attachment of the LEV. Our studies show that the downwash can lower the lift production approximately by $17 \%$ at hawkmoth's hovering Reynolds number, and by $22 \%$ at fruit fly's hovering Reynolds number; the difference seems less than substantial.

Examining from the established unsteady aerodynamic viewpoint, the LEV as a lift enhancement mechanism may be questionable because a dynamic-stall vortex on an airfoil is often found to break away and to convect elsewhere as soon as the wing translates [14]. The literature on helicopter blade models have been used to help explain the flapping wing aerodynamics; however, spanwise axial flows are generally considered to play a minor role in influencing the helicopter aerodynamics $[15,16]$. In particular, the helicopter blades operate at substantially higher Reynolds number and lower angle of attack. The much larger aspect ratio of a blade also makes the LEV harder to anchor. This is a key difference between helicopter blades and typical biological wings.

Employing three-dimensional Navier-Stokes computations [1,17], we show that the LEV is common to the flapping wing aerodynamics at Re (based on characteristic chord and flapping speed) of $\mathrm{O}\left(10^{4}\right)$ or lower, which corresponds to the insect flight regime. However, the LEV's main characteristics and the implications on lift generation change as $\operatorname{Re}$ (wing sizing, flapping frequency) varies. Figure 10 shows the streamline patterns at three Reynolds numbers; Figure 10(a) corresponds to a hawkmoth hovering at $\mathrm{Re}=6000$, 
Figure 10 (b) corresponds to a fruit fly at $\mathrm{Re}=120$, and Figure 10 (c) corresponds to a thrips at $\mathrm{Re}=10$. At $\mathrm{Re}=6000$, an intense, conical $\mathrm{LEV}$ core is observed on the paired wings with a substantial spanwise flow at the vortex core, breaking down at approximately three-quarters of the span towards the tip. At $\mathrm{Re}=120$ (Figure $10(\mathrm{~b})$ ), the vortex no longer breaks down and is connected to the tip vortex. The spanwise flow at the vortex core becomes weaker as the Reynolds number is lowered. Further reducing the Reynolds number to $\mathrm{Re}=10$, a vortex ring connecting the LEV, the tip vortex, and the trailing vortex is observed (Figure 10(c)); the flow structure shows more of a cylindrical than a conical form. Inspecting the momentum equation, one can see that the pressuregradient, the centrifugal force, and the Coriolis force together are likely to be responsible for the LEV stability.

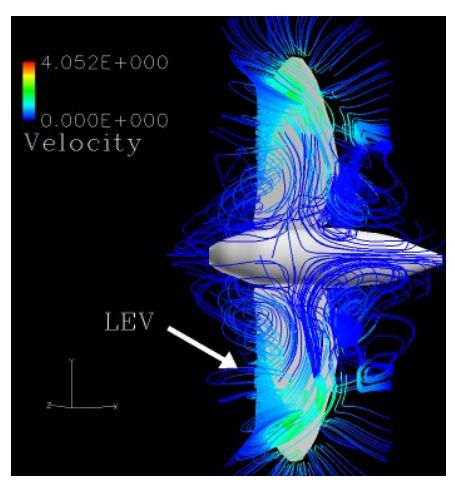

(a) $R e=6000$

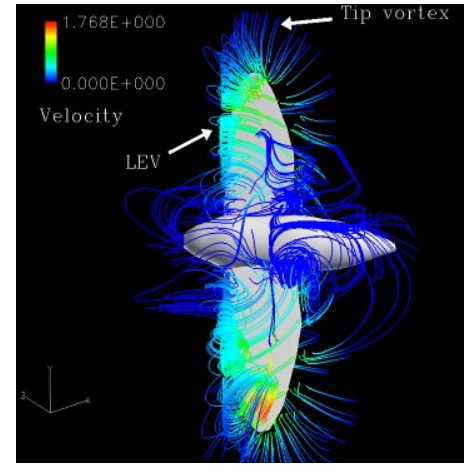

(b) $R e=120$

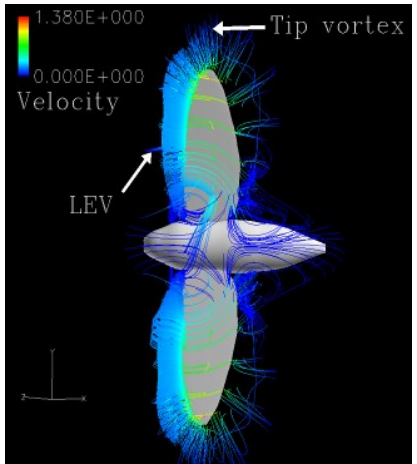

(c) $R e=10$

Figure 10. Streamlines and vorticity patterns asscoaited with the LEV at different Reynolds numbers.

The flow structures shown in Figure 10 are consistent with those reported experimentally. To further identify the roles of the translational and rotational motions of a flapping wing in the formation of the LEV, computed velocity vector distributions on an end-view plane, at $60 \%$ of wing span for $\mathrm{Re}=6000$ (hawkmoth) are compared against those for $\mathrm{Re}=134$ (fruit fly) in Figure 11 (a) and (b). The influence of wing rotation on the LEV is more evident at lower Re (134) than at the higher one (6000). On the other hand, the higher $\operatorname{Re}(6000)$ yields much more pronounced axial flow at the core of the LEV, which together with the LEV forms a helical flow structure near the leading edge. In contrast, only very weak axial flow is detected for the lower Re (134). Figure 11 (c) and (d) illustrates the pressure gradient contours on the wing of a fruit fly model and a hawkmoth model, respectively. Compared to hawkmoths, fruit flies, at a Re of $100 \sim 250$, cannot create as steep pressures gradient at the vortex core; nevertheless, they seem to be able to maintain a stable LEV during most of the down and upstroke. While the LEV on a hovering hawkmoth's wing breaks down in the middle of the downstroke, the LEV on the hovering fruit fly's wing stays attached during the entire downstroke, eventually breaking down during the subsequent supination. A weaker swirling flow tends to break down at a higher Reynolds number. Since the fruit fly exhibits a weaker LEV, from this viewpoint, it tends to better maintain the vortex structure than a hawkmoth, which creates a stronger 
LEV. Of course, the link regarding the vortex breakdown between a fixed and a flapping wing, if any, needs to be systematically investigated.

\section{A. $\mathrm{Re}=6000 \quad$ (Hawkmoth)}

(a)

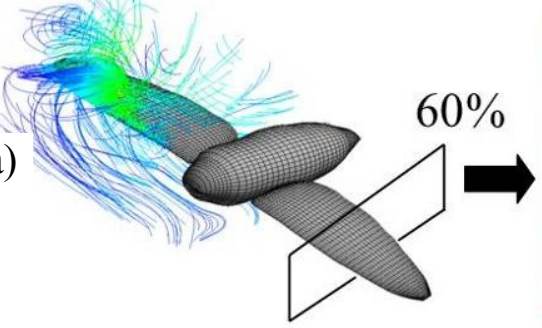

B. $\operatorname{Re}=134$ (Fruit fly)

(b)
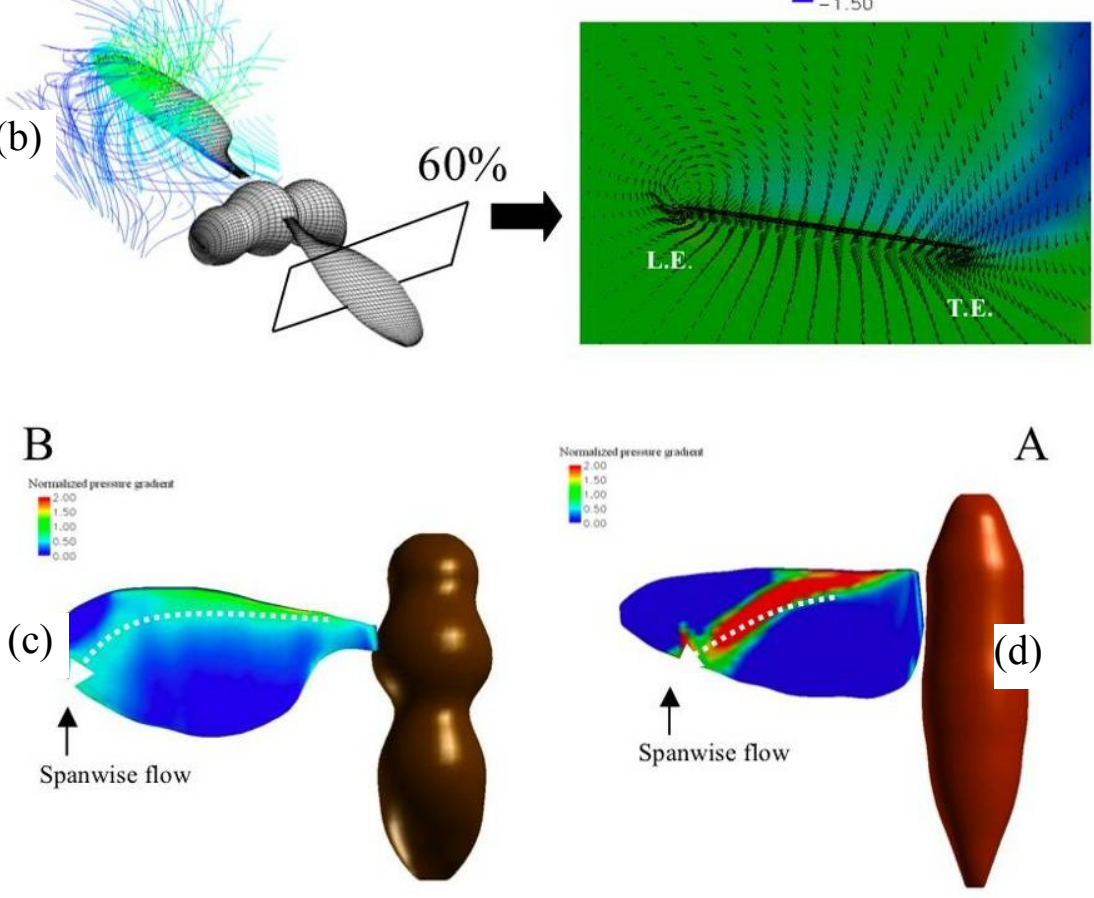

Figure 11. Comparison of near-field flow fields between a fruit fly and a hawkmoth. Wing-body computational model of (a) a hawkmoth $(\mathrm{Re}=6000)$, and (b) a fruit fly model $(\mathrm{Re}=134)$, with the LEVs visualized by instantaneous streamlines and the corresponding velocity vectors in a plane cutting through the left wing at $60 \%$ of the wing length; pressure gradient contours on the wing surface for (c) a fruit fly; and (d) a hawkmoth. The pressure gradient indicates the direction of the spanwise flow.

Vortex dynamics and wake topology in the near and far fields of a representative fruitfly are illustrated in Figure 12. There, a sequence of four iso-vorticity surface plots is presented, highlighting the formation and development of the flow structures in a complete stroke cycle. For the fruit fly, realistic wing-body kinematics turns out to solve the hovering problem in a similar way: to create a pair of horseshoe vortices (HSV) from tip, leading and trailing edge vortices and to evolve it into a vortex tube ring (VTR) pair 
with two jets present in its cores forming the hovering downwash (Figure 12). The aerodynamics of insect flapping flight is very three-dimensional, with multiple physical mechanisms utilized simultaneously. It is important to model the realistic wing-body morphology and kinematics to gain understanding of these low Reynolds flyers.
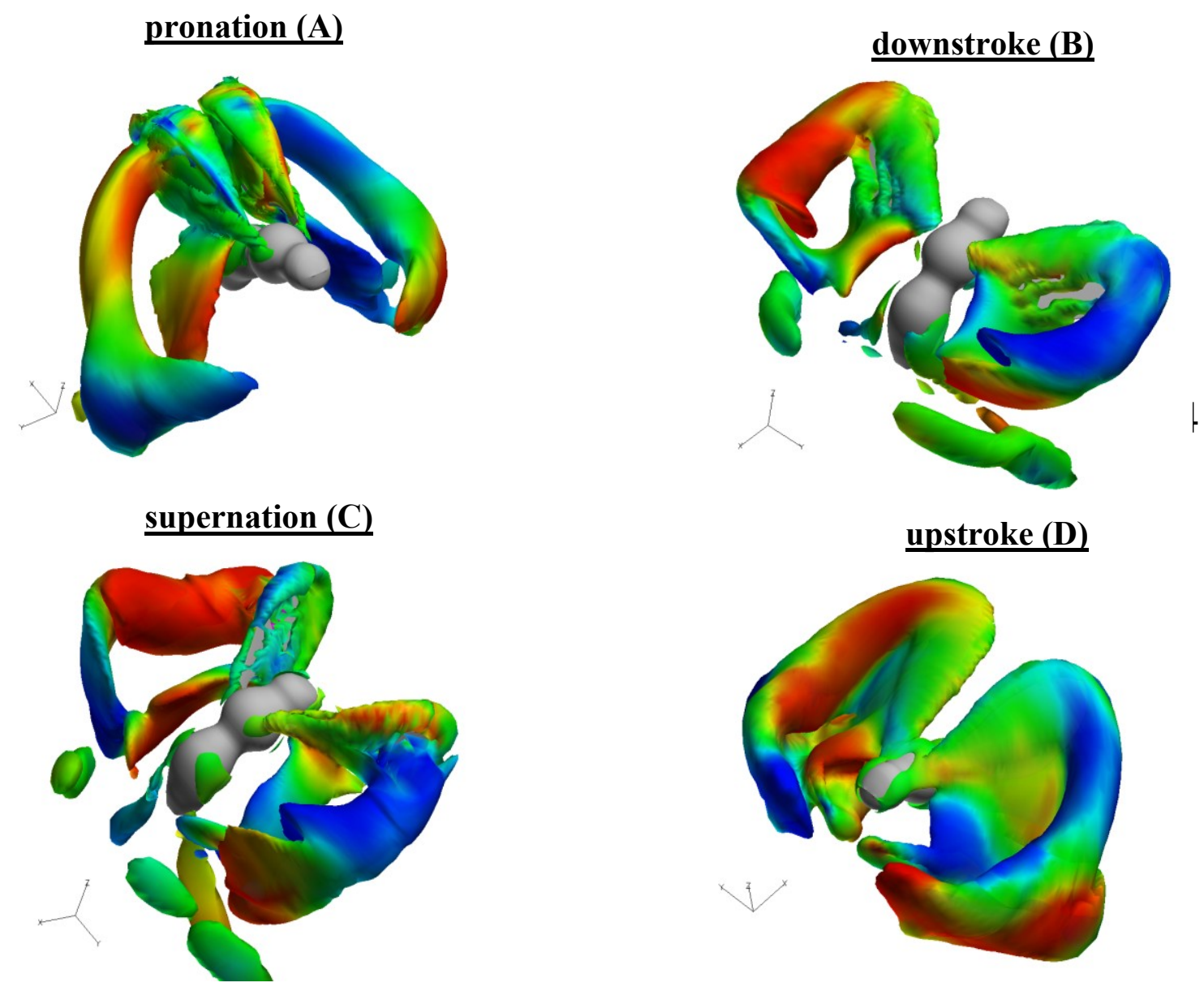

Figure 12.Visualization of flow fields around a hovering fruitfly. Absolute iso-vorticity surfaces around a hovering fruitfly during (A) the pronation, (B) the downstroke, (C) the supination and (D) the upstroke, respectively. The color of iso-vorticity surfaces indicates the normalized helicity density which is defined as the projection of a fluid's spin vector in the direction of its momentum vector, being positive (red) if it points in the same direction and negative (blue) if it points in the opposite direction.

\section{(ii) Aerodynamics \& Flapping kinematics}

It is known that depending on the flapping wing kinematics, stroke amplitude, frequency, Reynolds number, and the freestream environment, different flow structures result. Clearly, flapping kinematics has a strong influence on the time dependent aerodynamics, resulting in a variety of patterns and characteristics. For example, we have investigated two flapping modes, termed "water treading" (the airfoil is horizontally placed at the end of each half-stroke) and "normal" modes (the airfoil is vertically placed at the end of each 
half-stroke), as illustrated in Figure 13. They both are governed by the following simple stroke and AoA process during a single flapping cycle:

$$
h(t)=h_{a} \cos \left(2 \pi f t+\phi_{h}\right) \quad \alpha(t)=\alpha_{0}+\alpha_{a} \cos \left(\omega t+\phi_{\theta}\right)
$$
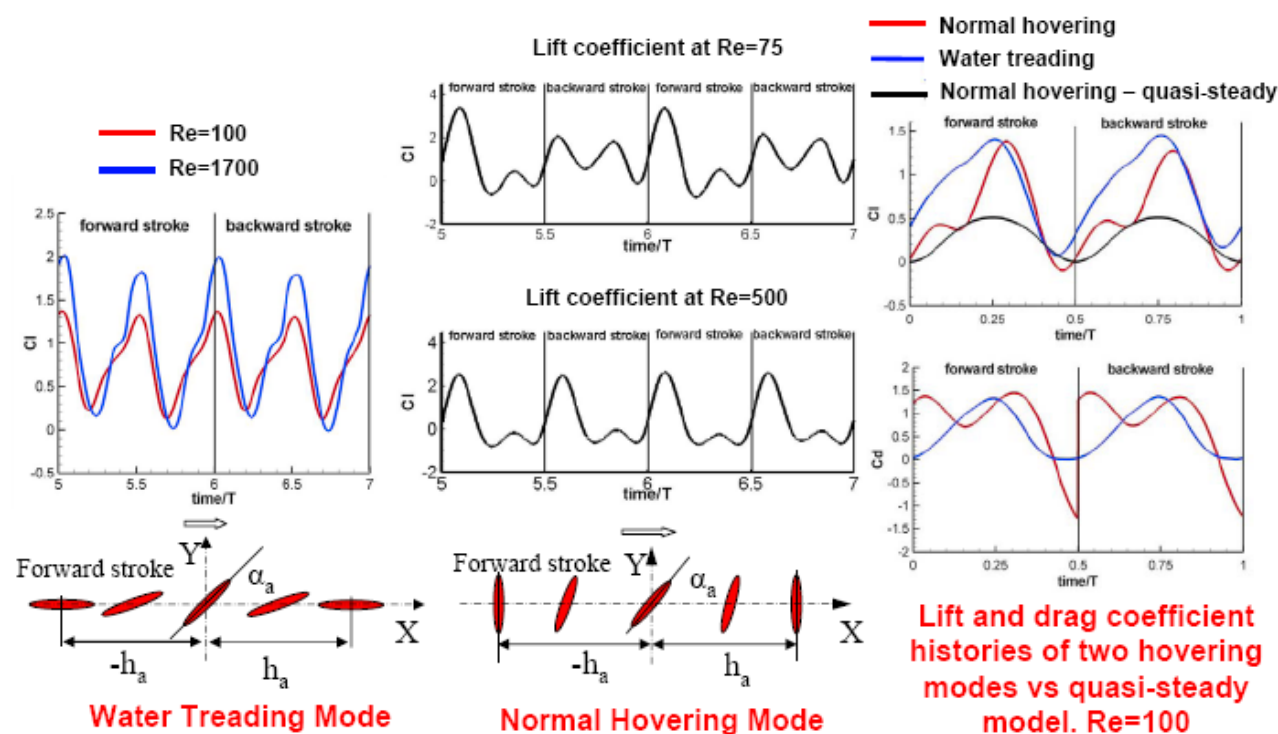

Figure 13. Interplay of kinematics and Re on lift generation of a flapping wing, and comparison between time dependent and quasi-steady model prediction of lift (upper right)

As highlighted in Figure 13, distinctly different aerodynamic performance is observed (i) between the two modes at the same Reynolds number, and (ii) with the same mode but between different Reynolds numbers. At a sufficiently low Reynolds number, both modes produce symmetric lift between the forward and backward strokes. However, substantially more asymmetric patterns are observed in the normal mode at higher Re. The literature has identified Weis-Fogh's clap-and-fling, leading-edge vortices, pitchingup rotation, and wake-capturing as noticeable mechanisms contributing to lift enhancement $[18,19,20,21]$. These findings indicate that in order to develop suitable knowledge base and design guidelines for flapping wing MAVs, a thorough understanding of the kinematics, large vortex structures and Reynolds number is essential since these processes directly influence the lift and thrust generation.

Efforts are being made in addressing the collective influence of various kinematic parameters on the aerodynamics of a flapping airfoil, including lift generation and power requirement. As a first step, we consider an ellipse with $15 \%$ thickness governed by equations illustrated in Figure 14. The three variables under consideration, the plunging amplitude $\left(h_{a}\right)$, the angular amplitude $\left(\alpha_{a}\right)$, and the phase lag $(\phi)$ between the translation and rotation, are systematically probed at a fixed Reynolds number of 100 with the aid of surrogate modeling techniques. The other variables in the equations are the frequency of translation/rotation $(f)$, time $(\mathrm{t})$, and mean angle of attack $\left(\alpha_{0}\right)$ which equals 90 for all of the cases under consideration. Instantaneous force histories coupled with the time 
averaged lift and power from the surrogate models provide insight into the impact of the kinematic parameters and the unsteady flight mechanisms.
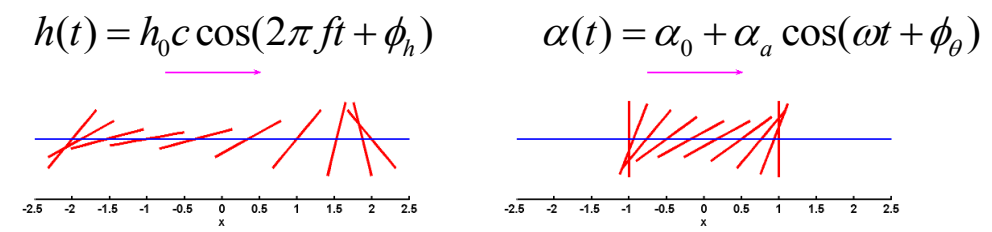

Figure 14. Example schematics for various parameter combinations. Left: $h_{a}=2.0, \alpha_{a}=80^{\circ}$, $\phi=210^{\circ}$ (advanced rotation) .Right: $h_{a}=1.0, \alpha_{a}=62.5^{\circ}, \phi=90^{\circ}$ (typical normal hovering).

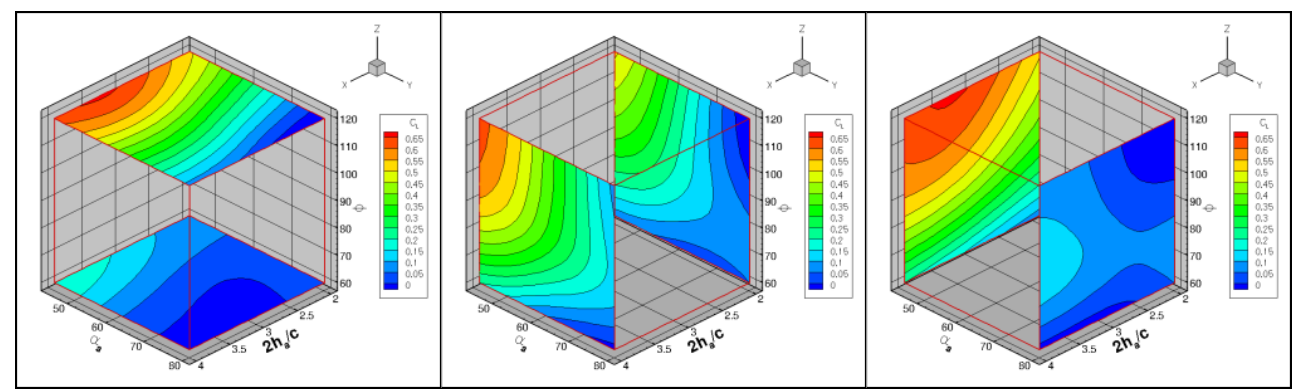

Figure 15. Time averaged $\mathrm{C}_{\mathrm{L}}$ as calculated from the surrogate models. The largest time averaged $C_{L}$ values obtained occur near $h_{a}=1.7, \alpha_{0}=0, \alpha_{a}=45^{\circ}, \phi=210^{\circ}$.

As illustrated in Figure 15 and concluded from a global sensitivity analysis, the plunging amplitude to chord ratio is the least influential design variable under consideration as well as the least coupled (statements which are also valid for the reduced frequency). While it appears that the net lift can be governed by a relatively few simple statements, e.g. lower angular amplitudes correlates to higher lift coefficients, as seen below there are more complex interactions which are somewhat lost in the integrated result.

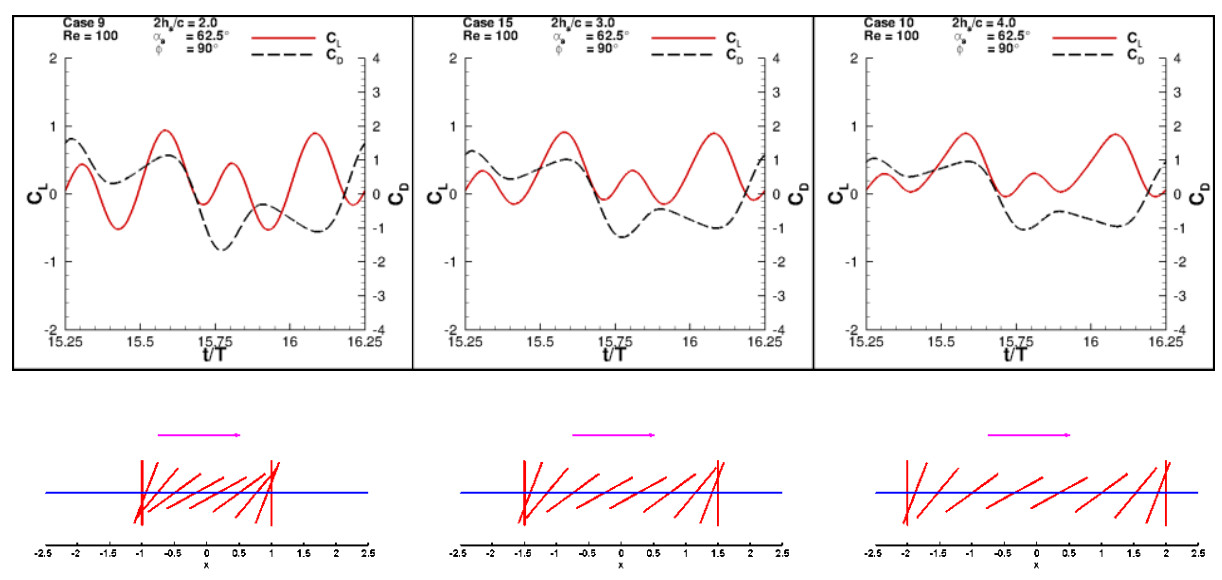

Figure 16. Force histories over one cycle showing the effect of increasing the plunging amplitude to chord ratio (or lowering the reduced frequency). Competition of effects is seen between the wake capturing peaks and subsequent valleys. The time averaged lift coefficient $<\mathrm{C}_{\mathrm{L}}>$ from left to right: $0.18,0.28,0.34$. 


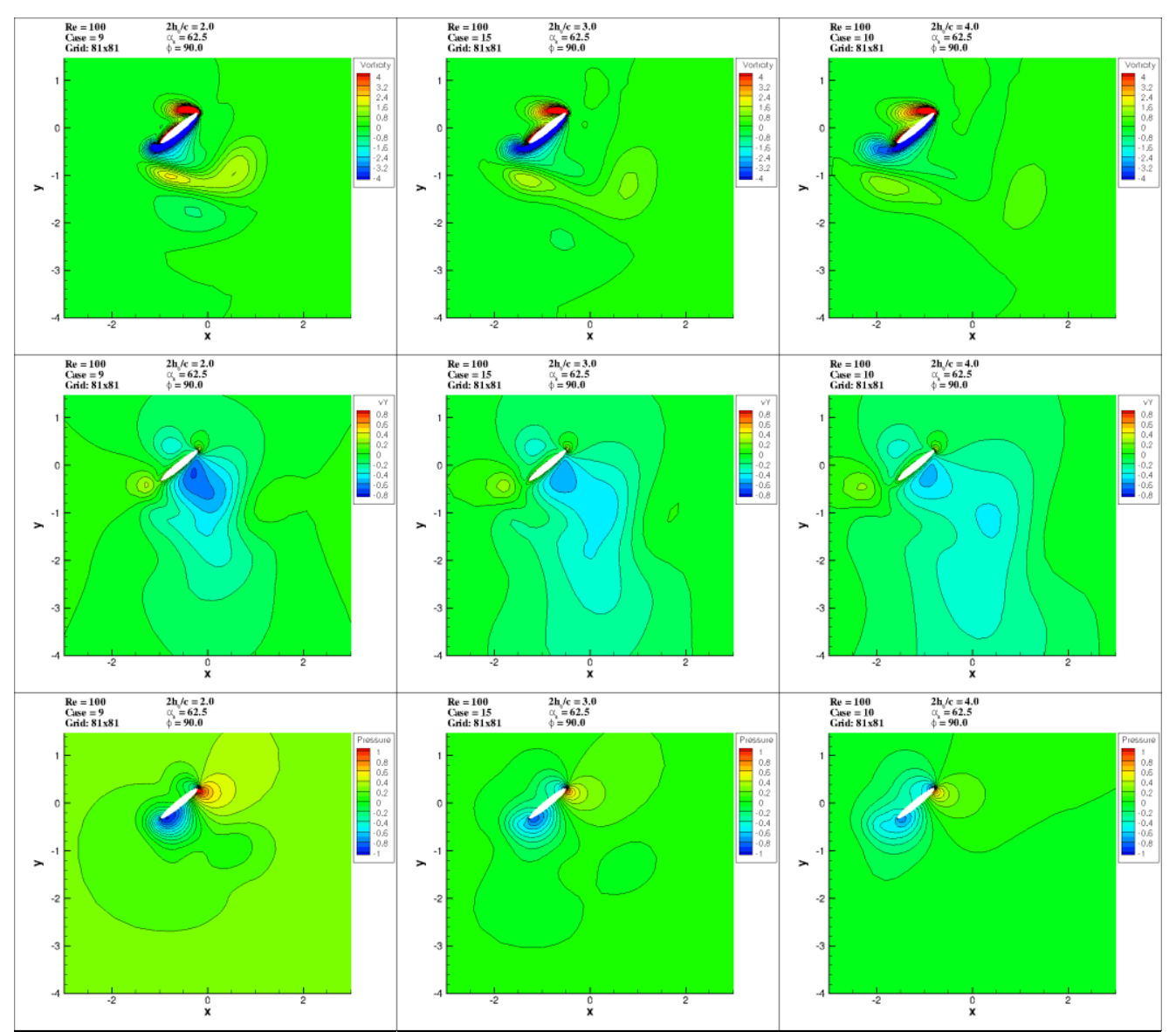

Figure 17. Flow field shots at $\mathrm{t} / \mathrm{T}=15.9$ illustrating vorticity (top), v-velocity (middle), and pressure (bottom) during the wake capture valley. The less intense interaction, larger plunging amplitude to chord ratio, yields better lift results.
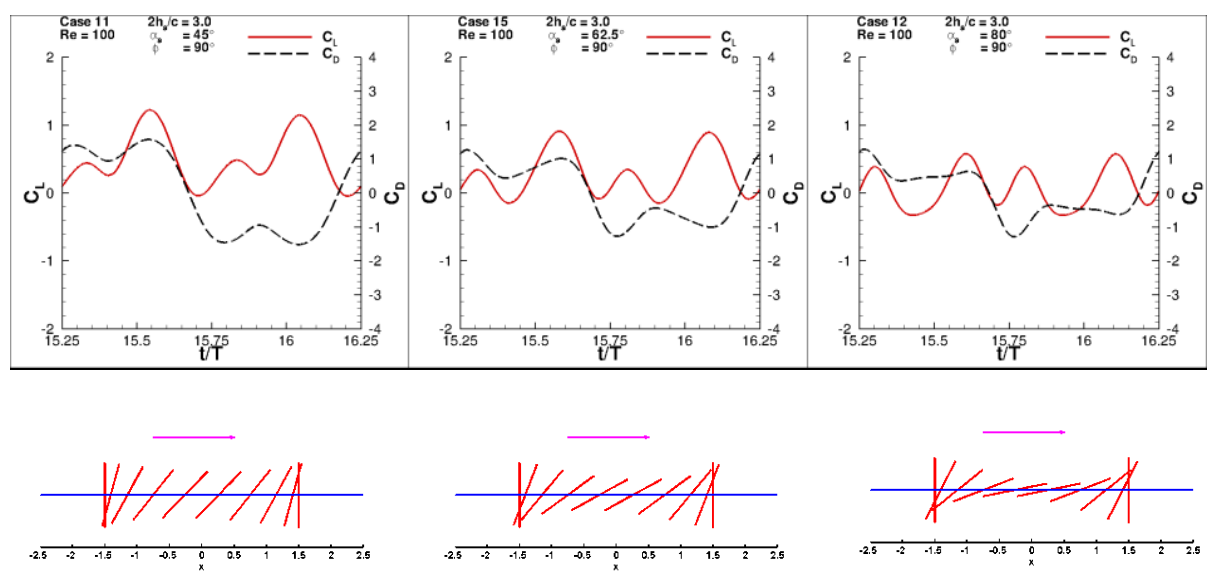

Figure 18. Force histories over one cycle showing the effect of changing the angular amplitude. The consequences of higher angular amplitudes are detrimental to the wake capturing valley as well as the delayed stall peak. The time averaged lift coefficient $\left\langle\mathrm{C}_{\mathrm{L}}>\right.$ from left to right as the angular amplitude increases: $0.50,0.28,0.07$. 


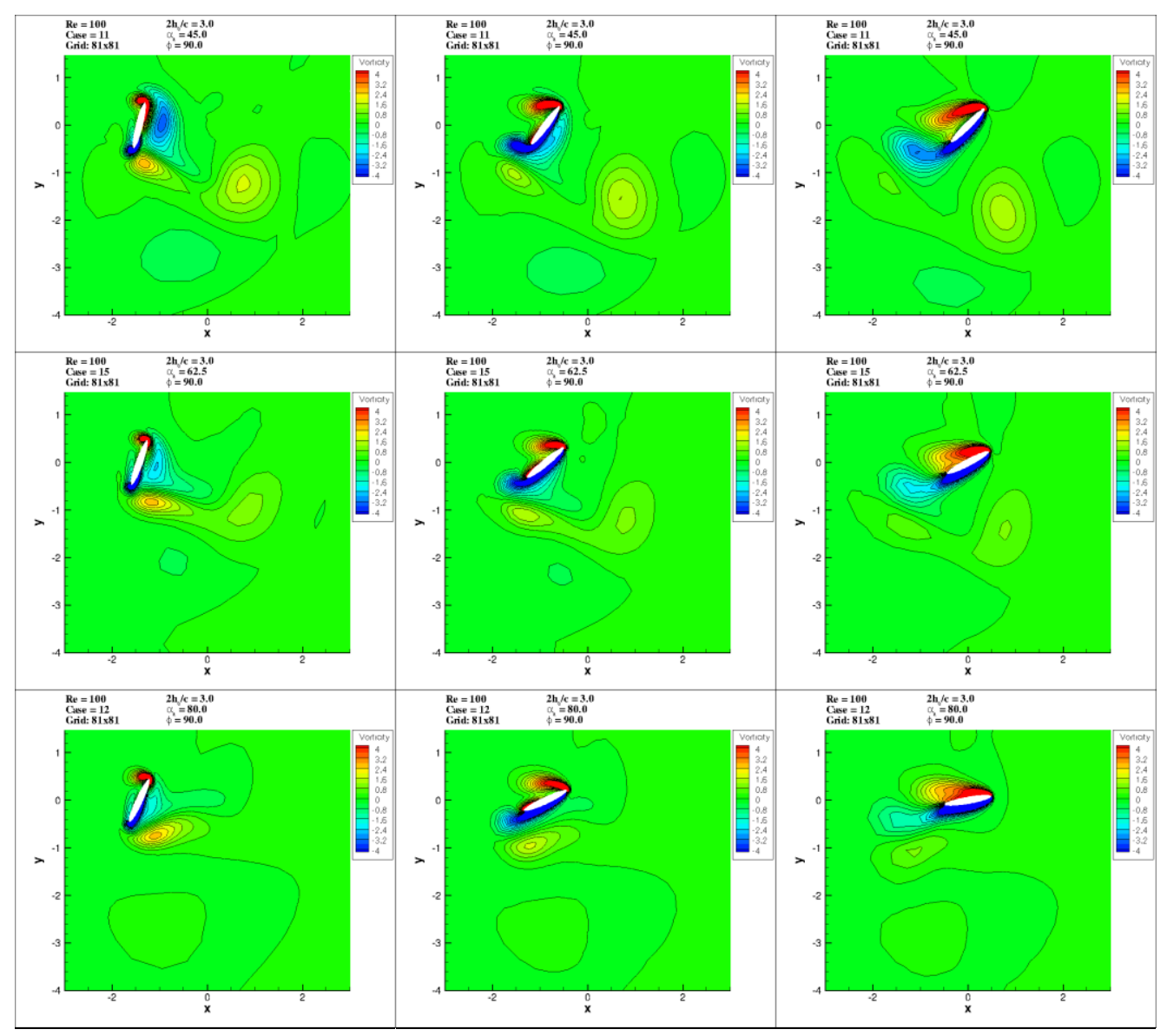

Figure 19. Flow field shots of the vorticity at $\mathrm{t} / \mathrm{T}=15.8$ (wake capture peak), 15.9 (wake capture valley), 16.0 (delayed stall peak) for $\alpha_{a}=45^{\circ}$ (top), $\alpha_{a}=62.5^{\circ}$ (middle), and $\alpha_{a}=$ $80^{\circ}$ (bottom).

In the context of the regime studied, it is found that: (i) The delayed stall mechanism interacting with the wake capture makes the primary lift contribution. Higher angular amplitudes (lower angles of attack) may not allow for leading edge vortex formation. (ii) The wake capturing peak is not as sensitive as the subsequent valley to changes in the design variable. This valley is caused by interaction with a jet-like flow feature which accelerates the flow on the underside of the airfoil leading to lower pressures/lift and greatly influences the integrated lift values. (iii) The plunging amplitude/ reduced frequency is the least influential parameter by a significant margin when examining the lift coefficient. (iv) Advanced rotation $\left(\phi>90^{\circ}\right)$ changes the phase between the translation and rotation such that at the peak velocity translational velocity, higher angles of attack are achieved while the angular velocity is positive. In contrast during strict normal hovering $\left(\phi=90^{\circ}\right.$ ), the lowest angle of attack occurs during the peak translational velocity at which point the angular velocity is equal to zero. 
(iii)Laminar-turbulent transition, wind gust, and multiple time scales

For flapping wings operating at Reynolds number of $\mathrm{O}\left(10^{4}\right)$ or lower, the flow may experience transition from laminar to turbulent. Several obvious scenarios include (i) the classical Tollmien-Schlichting wave where small disturbances grow progressively as the fluid travels downstream, eventually becoming turbulent, (ii) vortex breakdown instabilities of leading edge vortices, and (iii) bypass transition where the disturbance is large and the flow transitions to the turbulent state without going through the disturbance growth process. It is known that the drag-polar and lift curve slope of airfoils at $\operatorname{Re}<10^{5}$ are noticeably affected by laminar separation, exhibiting the so-called drag bulge. Efforts are being made experimentally and computationally to investigate low Reynolds number transition [22, 23, 24, 25, 26]. Transition and leading edge vortex breakdown in biological flyers have not been adequately studied. It is most important to extend our knowledge from 2D airfoil investigations to 3D low aspect ratio wings and from laboratory conditions to complex gusty conditions capturing transition phenomena at the Reynolds numbers of interest.

Figure 20 compares the experimental measurement by Radespiel et al. and our simulation of flow over SD7003 airfoil at Reynolds number of 60,000 and AoA of 4 degrees. Our results show that flow experiences transition at $50 \%$ of the chord position from the leading edge while the experiment shows transition occurs at $55 \%$ of the chord. It should be noted that in the experiment, the transition location is defined as the point where the normalized Reynolds shear stress reaches $0.1 \%$ and demonstrates a clearly visible rise. The transition point in our simulation is defined as the point where the most unstable TS wave has amplified by a factor of $\mathrm{e}^{N}$. If using the normalized shear stress threshold $0.1 \%$ as the criteria, the transition point is at $56 \%$ of the chord based on our simulation. Overall, as shown in Figure 20, our simulation shows good agreement with the experimental results in terms of transition position, reattachment position, and vortex core position. However, our simulations have noticeably lower shear stress magnitude than the experiment.

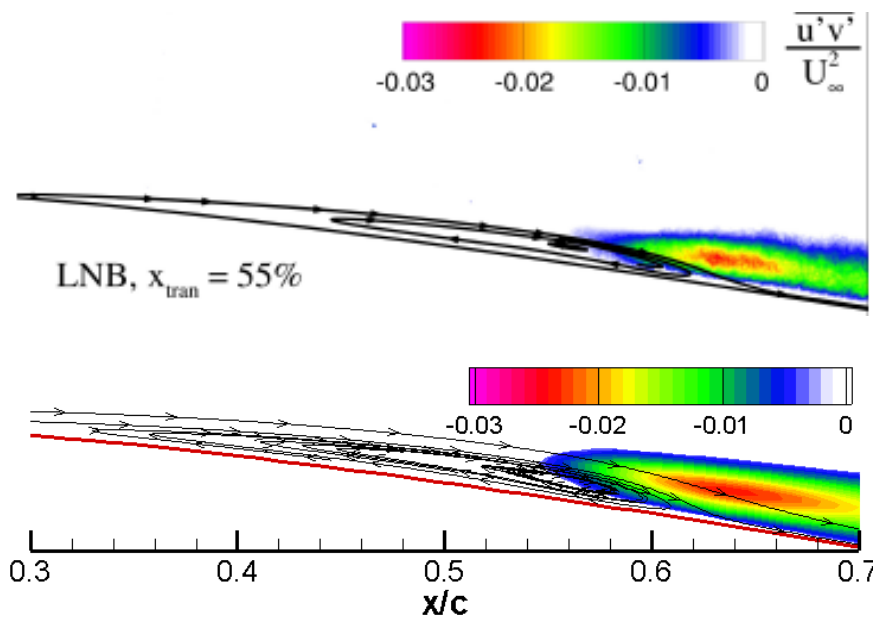

Figure 20. Streamlines and turbulent shear stress for $\alpha=4^{\circ}$. Top: experimental measurement by Radespiel et al.[25]; bottom: present numerical simulation with $N=8$. 
Wind gusts create unsteadiness in the flight environment. The effect of unsteadiness resulting from wind gusts, especially the crosswinds and updrafts that are anticipated in urban environments are even more destabilizing than head-on gusts. Their influence on vehicle performance and control response time must be accounted for. Fundamentally, the characteristic flapping time scale of insects and hummingbird (tens to hundreds of $\mathrm{Hz}$ ) is much shorter than the time scale of typical wind gust (around $1 \mathrm{~Hz}$, as discussed in Shyy et al. [27]). Hence, from the flapping wing time scale, many wind gust effects can be treated in a quasi-steady manner. However, the vehicle control system (as in the case of a biological flyer) operates at lower frequencies, and their time scales are comparable to that of anticipated wind gusts. Therefore, there is a clear multi-scale problem between unsteady aerodynamics, wind gust, and vehicle control dynamics. Figure 21 shows the computed lift and drag histories of a stationary NACA0012 airfoil in gusty environment by Lian and Shyy [7]. The Reynolds number is $4 \times 10^{4}$ based on the freestream velocity and chord length. The AoA is set to $4^{\circ}$. At this Reynolds number and AoA, laminar separation bubble causes flow to experience laminar to turbulent transition. The head-on gust with a single frequency of $1 \mathrm{~Hz}$ follows:

$$
U(t)=U_{0}\left(1+N_{A} \sin \left(\omega_{g} t\right)\right)
$$

where $\omega_{g}$ is the gust circular frequency, and $N_{A}$ is the fluctuation amplitude. Here $\omega_{g}$ is equal to $2 \pi$ and $N_{A}$ is 0.2 . Comparisons are also made with quasi-steady simulation. Overall, the quasi-steady simulation predicts higher lift and lower drag than the unsteady simulation at the same instantaneous Reynolds number. The lift curve of the quasi-steady simulation has similar pattern to that of the unsteady computation, both following the freestream velocity variation, $U^{2} / U_{0}^{2}$. On the other hand, the drag from the unsteady simulation is quite different from that from the quasi-steady prediction.

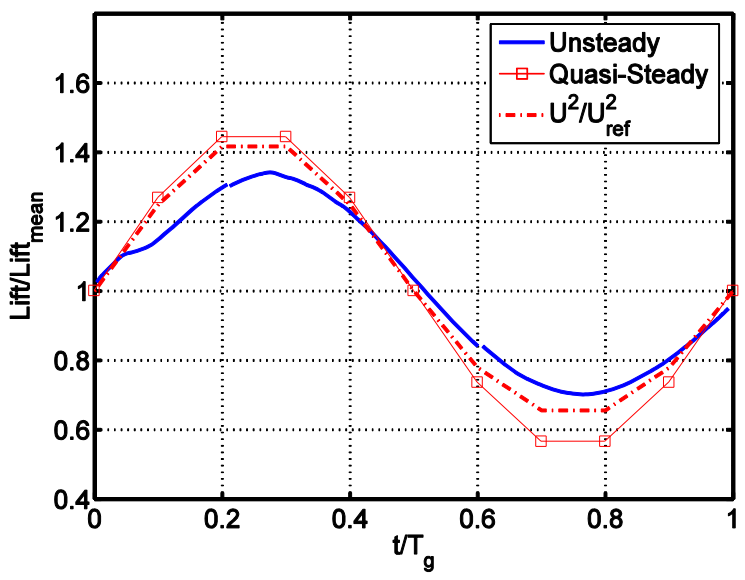

(a)

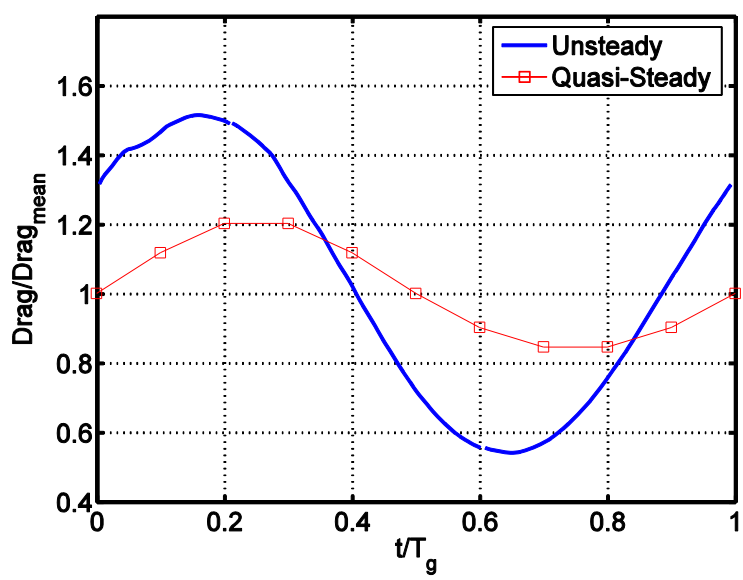

(b)

Figure 21. Comparison of lift and drag coefficients between unsteady and quasi-steady computations of a stationary airfoil. (a): lift coefficient; (b): drag coefficient. (Transitional flow simulation) 
Time histories of the thrust and lift coefficients and time-averaged thrust of a flapping NACA0012 airfoil are shown in Figure 21. The time is normalized by the gust period, $\mathrm{T}_{\mathrm{g}}$. We have the following observations. First, during each gust cycle, the thrust and lift coefficients changes in the opposite direction of the freestream velocity. There is a clear pattern that the maximum magnitudes of lift and thrust coefficients during each cycle decrease with the increase of freestream velocity and increase with the decrease of the freestream velocity. It looks like that there is a phase lag of $180^{\circ}$ between the force coefficient and the freestream velocity. Many factors can contribute to this phase lag, such as flapping kinematics and imposed gust. Second, the flapping motion acts like a filter that reduces the freestream variation. From Figure 22(c) we can see that the timeaveraged thrust (over each flapping cycle) has a variation of less than $10 \%$ over its mean value even though the freestream velocity has a $20 \%$ variation. This will help the vehicles using flapping wing maintain a stable thrust under gust environment.

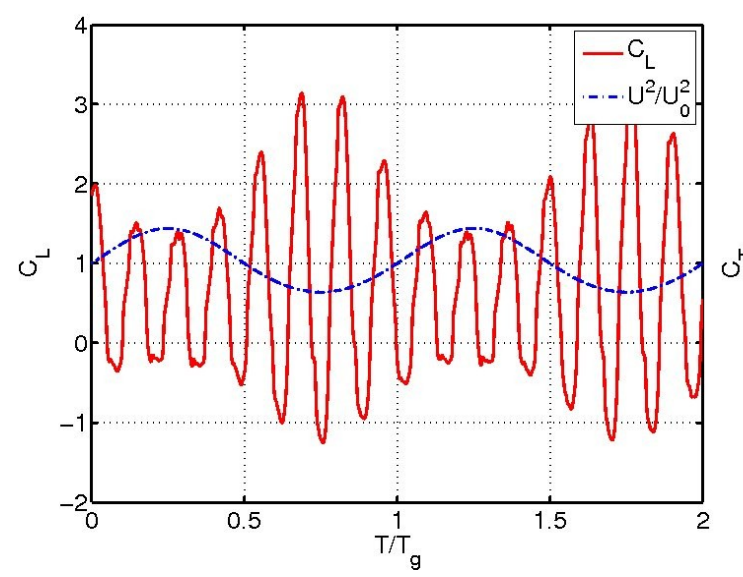

(a)

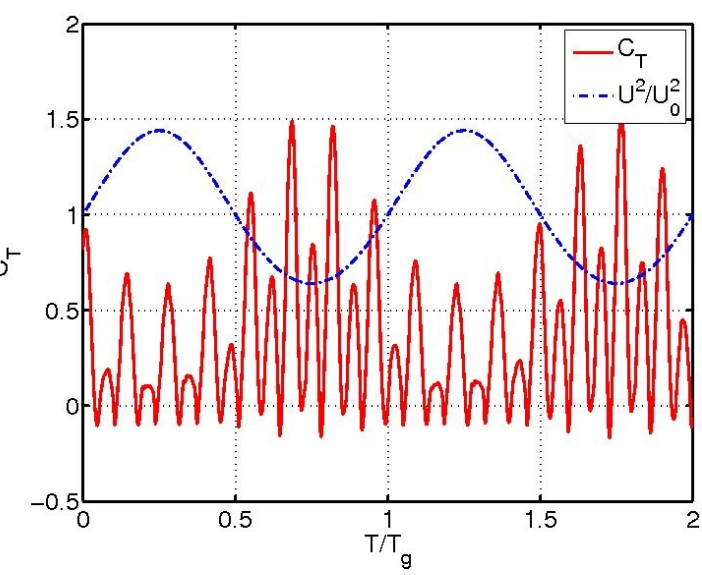

(b)

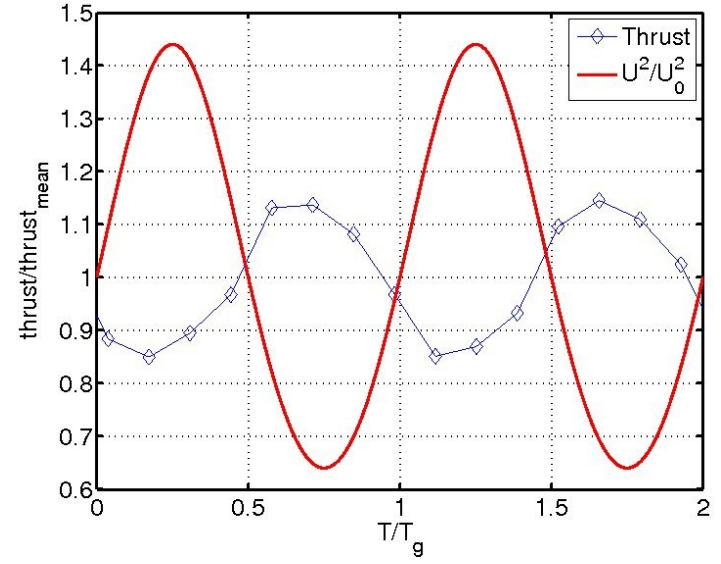

(c)

Figure 22. Force history of a flapping airfoil during one gust cycle. (a) Thrust coefficient history; (b) Lift coefficient history; (c) Time-averaged thrust. $\mathrm{Re}=4 \times 10^{4}, h_{\mathrm{a}}=0.75 \mathrm{c}, S t=$ $0.3, k=0.63, \varphi=\varphi_{\theta}-\varphi_{h}=75^{\circ}, \alpha_{0}=0$, and $\alpha_{0}=28^{\circ}$.(Laminar flow simulation). 


\section{(iv) Fluid-structure interactions and aerodynamics enhancement}

Our research has clearly established that local flexibility can delay stall and enhance aerodynamics in fixed wings [28]. Furthermore, as already discussed above, insect's wing properties are anisotropic, with the spanwise bending stiffness about 1 to 2 orders of magnitude larger than the chordwise bending one. In general, spanwise flexural stiffness scales with the third power of the wing chord while the chordwise stiffness scales with the second power of the wing chord [29]. Moreover, the thin nature of the skin structure makes it unsuitable for taking compressive loads, which may result in skin wrinkling and/or buckling (i.e., large local deformations that will interact with the flow). Can those large flexible deformations provide a better interaction with the aerodynamics than if it was limited to the linear regimes? If torsion stiffness can be tailored over the plane of the wing, how that can affect the wing kinematics for optimum thrust generation? But not only torsion is practiced in flapping wing locomotion. As shown in Fig. 3, both torsion and chordwise deformation are employed depending on the instantaneous flight condition and the control need. How do these geometrically nonlinear effects and the anisotropy of the structure impact the aerodynamics characteristics on the flapping wing? All these are issues that require detailed investigations and are critical for the success of future MAV designs.

Forward flight with flexible plunging airfoil

The flow field of a flexible, flapping flat plate is investigated by Tang et al. [31]. The Reynolds number based on the freestream velocity and the plate length is $R e=9000$. To solve the structural displacements, they adopt a 2D finite element method using beam elements. The displacement $y$ is given by the beam differential equation

$$
\rho_{s} b \frac{\partial^{2} y}{\partial t^{2}}+\frac{\partial^{2}}{\partial x^{2}}\left(E I \frac{\partial^{2} y}{\partial x^{2}}\right)=q
$$

where $\rho_{s}$ is the density of the beam, $b$ is the beam height, $E$ is the Young's modulus, $I$ is the area moment of inertia of the cross section of beam element, and $q$ is the distributed loading acting in the same direction of the displacement $y$. Nondimensionalizing by $c, \rho$, and $U$, we obtain

$$
\bar{\rho} \frac{b}{c} \frac{\partial^{2} \bar{y}}{\partial \bar{t}^{2}}+\overline{E I} \frac{\partial^{4} \bar{y}}{\partial \bar{x}^{4}}=\bar{q}
$$

where $\left(^{-}\right)$denotes the non-dimensionalized variables. The non-dimensional parameters, density $\bar{\rho}$, modulus of elasticity $\bar{E}$ and inertia area moment of the beam cross section $\bar{I}$, are defined as 


$$
\begin{aligned}
& \bar{\rho}=\frac{\rho_{s}}{\rho_{f}} \\
& \bar{E}=\frac{E}{\rho_{f} U^{2}} \\
& \bar{I}=\frac{I}{c^{3}}
\end{aligned}
$$

For a flat plate with thickness $b$ and unit width, the area moment of inertia is $I=1 / 12 \cdot b^{3}$. Then the bending rigidity becomes

$$
\bar{I}=\frac{1}{12}\left(\frac{b}{c}\right)^{3}
$$

Since the Young's modulus $E$, the densities $\rho_{f}, \rho_{s}$ and velocity scale $U$ are considered to be constant, a representative non-dimensional parameter is the thickness to chord ratio $b / c$. Non-dimensional material properties adopted by Tang et al. [31] are as follows: modulus of elasticity $2.05 \times 10^{10}$, density 7.85 . The stiffness varies by changing the thickness of the plate. Three plates with relative thickness of $b / c=0.56 \times 10^{-3}, b / c=1.41 \times 10^{-}$ ${ }^{3}, \mathrm{~b} / \mathrm{c}=4.23 \times 10^{-3}$ are used in present research. A schematic of the plunging displacement $h(t)$ and pitching angle $\alpha(t)$ for the plunging motion mode are shown inFigure 23 . The plunging and pitching of the upward- and downward-strokes in each cycle are the sinusoidal function. The incoming flow is along the horizontal direction. Specifically, the plunging and pitching amplitudes are:

$$
\begin{aligned}
& h(t)=h_{a} \sin (2 \pi f t) \\
& \alpha(t)=\alpha_{0}+\alpha_{a} \sin (2 \pi f t+\varphi)
\end{aligned}
$$

where $\alpha_{a}$ is the pitching amplitude, $h_{a}$ is the dimensionless plunging amplitude normalized by the chord length $c, \alpha_{0}$ is the initial positional angle of the airfoil, and $\varphi$ is the phase difference between the plunging and pitching motion. Also, $h(t)=y_{L E}(t) / c$ where $y_{L E}$ is the vertical coordinate of the leading edge of the airfoil. In the present investigation, two kinematic patterns are considered, namely, (i) forward flight with pure plunging and no pitching, and (ii) forward flight with combined plunging with pitching. The airfoil performs plunging motion without pitching $\left(\alpha_{a}=0, \alpha_{0}=0\right)$. The plunging amplitude is $h_{a}=0.194$, normalized by the chord length $c$, Strouhal number is 1.4 , and $\varphi=\pi / 2$. Differences between the leading and trailing edge's vertical coordinates, together with the position of the leading edge are presented in Figure 24. The results indicate that deformation not only increase with the flexibility of the plate, which is obvious, but also creates a phase difference relative to the pitching motion. To characterize the deformation of the airfoil, an equivalent pitching angle is adopted, which is defined as the pitching angle of a rigid airfoil with same leading and trailing edge positions of the flexible airfoil. Considering that the length of plates does not change, the vertical coordinates' difference reflects the equivalent pitching angle. Figure 24 indicates 
that the equivalent pitching motion of a flexible plate $\left(\right.$ LE-TE, $\left.\mathrm{b} / \mathrm{c}=0.56 \times 10^{-3}\right)$ leads the plunging motion (position history of leading edge). This observation agrees with the experimental work of Heathcote and Gursul [30].

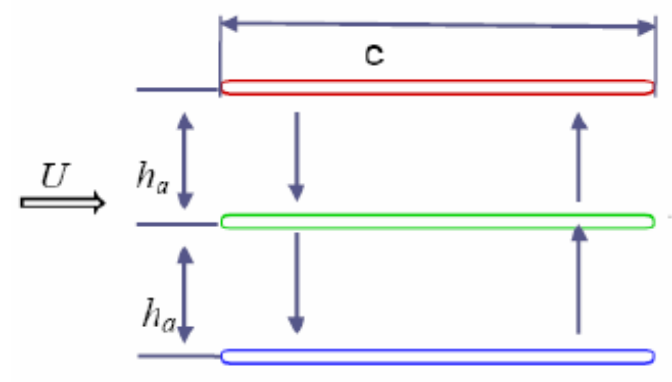

(i) plunging mode

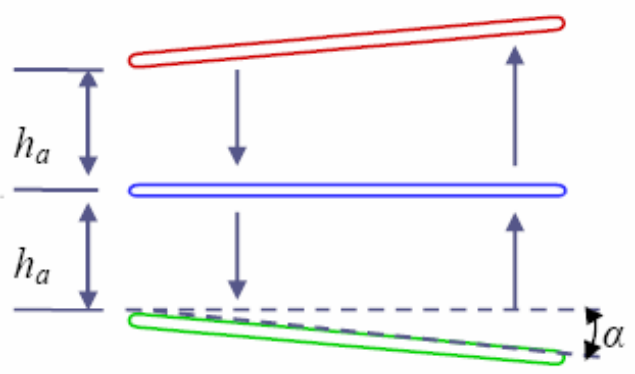

(ii) plunging and pitching mode

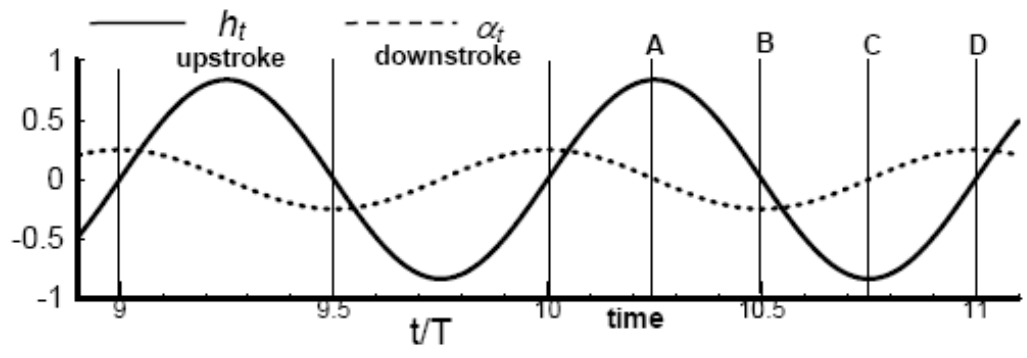

(iii) time history of plunging and pitching motion

Figure 23. Illustration of the flapping motion. (i. plunging mode; ii. plunging and pitching mode; iii. time history of pitching and plunging motion).

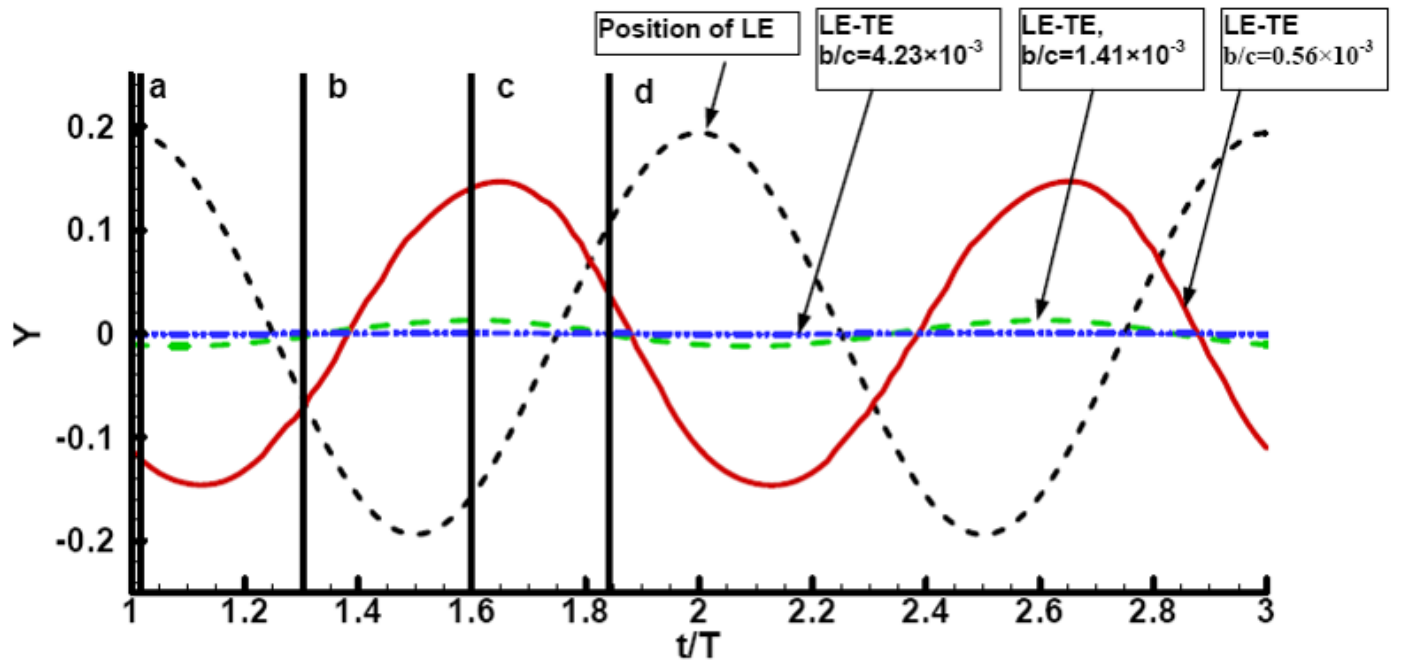

Figure 24. Time history of displacement differences of LE and TE coordinate of a plunging flexible plate $\left(\mathrm{Re}=9000, S t=1.4, \mathrm{~h}_{\mathrm{a}}=0.194, \mathrm{~b} / \mathrm{c}=0.56 \times 10^{-3}, \mathrm{~b} / \mathrm{c}=1.41 \times 10^{-3}\right.$, $\mathrm{b} / \mathrm{c}=4.23 \times 10^{-3}$. LE: leading edge, TE: trailing edge). 


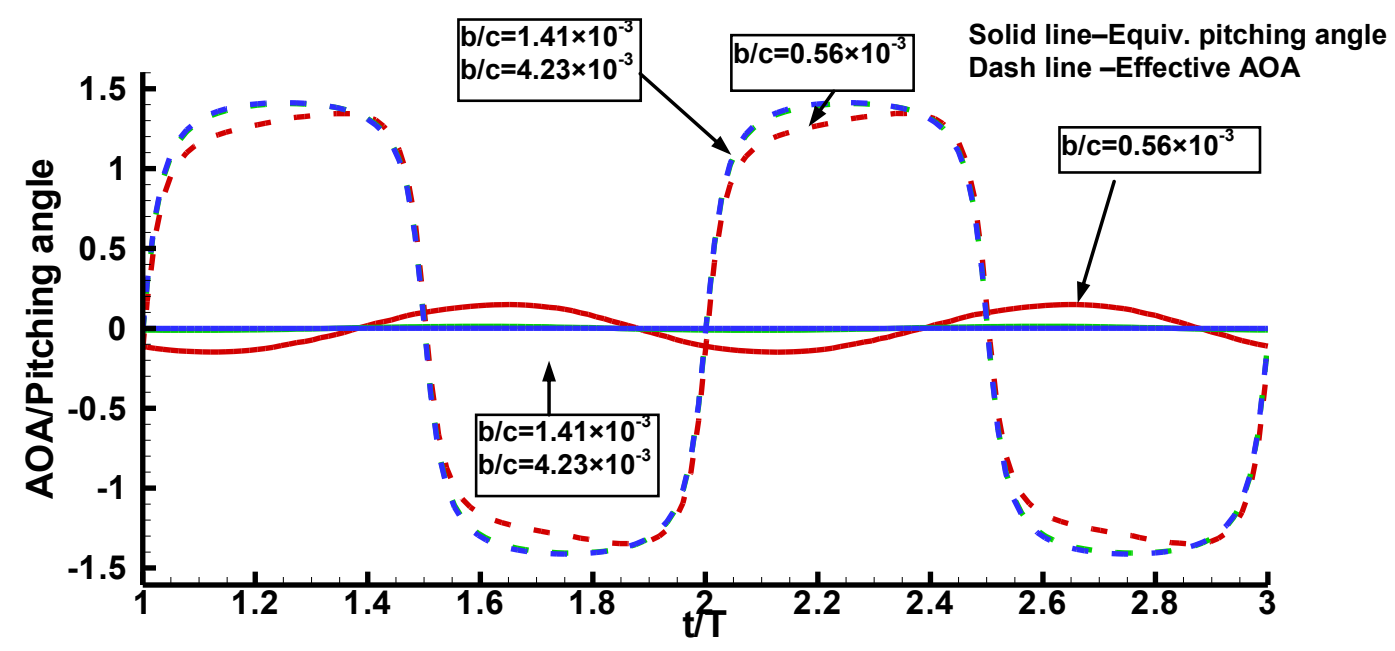

Figure 25. Time history of equivalent plunging angle and effective AoA ( $\mathrm{Re}=9000$, $\left.S t=1.4, \mathrm{~h}_{\mathrm{a}}=0.194, \mathrm{~b} / \mathrm{c}=0.56 \times 10^{-3}, \mathrm{~b} / \mathrm{c}=1.41 \times 10^{-3}, \mathrm{~b} / \mathrm{c}=4.23 \times 10^{-3}\right)$.
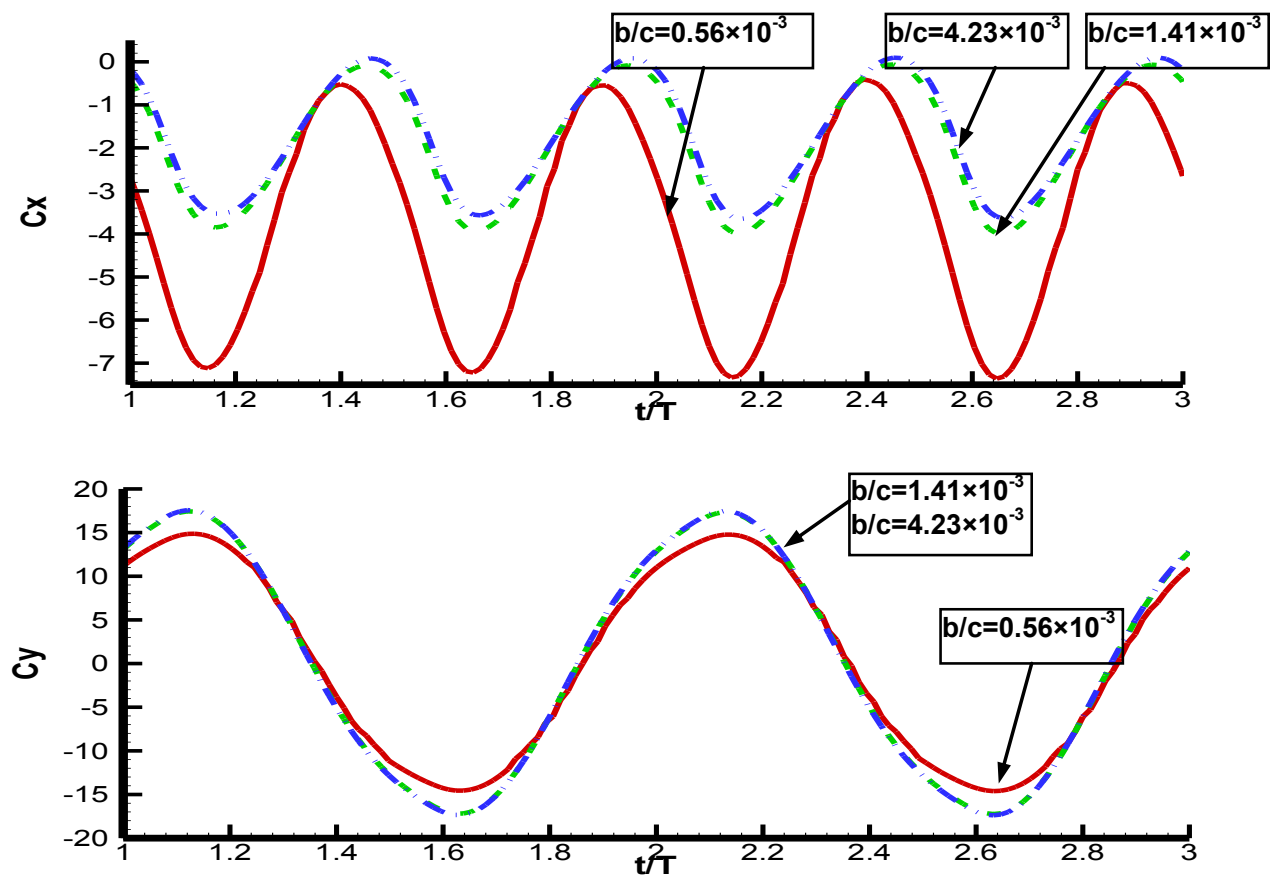

Figure 26. Time history of coefficients of horizontal and vertical force $(\mathrm{Re}=9000, S t=1.4$, $\mathrm{h}_{\mathrm{a}}=0.194$, and three thicknesses: $\left.\mathrm{b} / \mathrm{c}=0.56 \times 10^{-3}, \mathrm{~b} / \mathrm{c}=1.41 \times 10^{-3}, \mathrm{~b} / \mathrm{c}=4.23 \times 10^{-3}\right)$.

Figure 25 illustrates the effect of the equivalent plunging angle by presenting the effective AoA, which is based on the rigid airfoil's instantaneous position in accordance with the combined freestream and plunging motion, and equivalent pitching angle vs. 
time. It indicates that as the airfoil becomes more flexible, the effective angle of attack is reduced by the equivalent pitching motion. This means that for a flexible airfoil, the lift fluctuation during a flapping cycle is smaller than for a rigid airfoil. Similar observations have been reported for fixed wing, where a flexible structure can smooth out the fluctuation in lift in a gusty freestream. Figure 26 presents the time history of the horizontal (thrust) and vertical (lift) force coefficients. It shows that as the airfoil becomes more flexible, a higher thrust and, a smaller lift, is generated.

Figure 27 presents the streamlines, pressure contours and surface pressure distributions around the airfoil with different thicknesses at the time instants indicated in Figure 24. Overall, the fluid dynamics characteristics of all three cases are essentially the same. However, Figure 26 shows that the horizontal force (thrust) on the thinnest plate is much larger than the two stiffer plates. It seems that the pitching motion resulting from the airfoil shape flexibility impacts the equivalent angle of attack, causing different projected horizontal and vertical areas, and correspondingly, different lift and thrust coefficients.

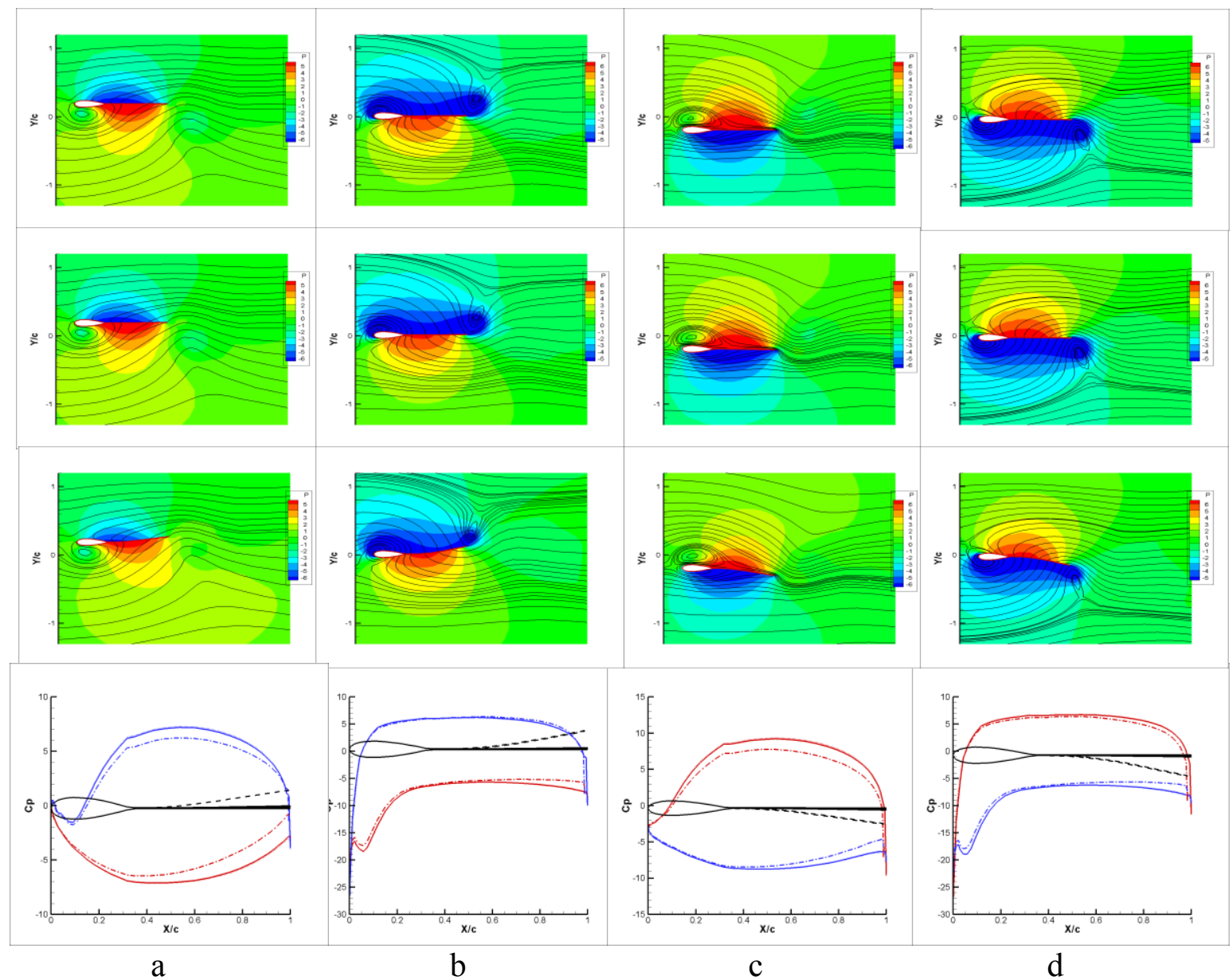

Figure 27. Flowfield of the numerical results of three thickness plates $(\mathrm{Re}=9000, S t=1.4$, $\mathrm{b} / \mathrm{c}=4.23 \times 10^{-3}$ (top), $\mathrm{b} / \mathrm{c}=1.41 \times 10^{-3}$ (middle), $\mathrm{b} / \mathrm{c}=0.56 \times 10^{-3}$ (lower)) and the pressure distribution on the airfoils (bottom) (in pressure distribution, solid lines (overlapped): $\mathrm{b} / \mathrm{c}=4.23 \times 10^{-3}, \mathrm{~b} / \mathrm{c}=1.41 \times 10^{-3}$; dash line: $\left.\mathrm{b} / \mathrm{c}=0.56 \times 10^{-3}\right)$. 
Membrane wing in steady flow

Passive shape adaptation can be built into a membrane wing through either geometric or aerodynamic twist. The former is a nose-down rotation of each wing section, wherein the streamlining alleviates maneuver/gust loads [32], and can delay the onset of stall. The latter is a rotation of the zero-lift angle of attack via load-induced membrane inflation, which can increase both the maximum lift coefficient and the longitudinal static stability, albeit with a drag penalty [33]. Successful implementation of passive shape adaptation is particularly important for micro air vehicles beset with several flight issues: poor wing efficiency due to separation of the low Reynolds number flow, rolling instabilities and bilateral asymmetries due to destabilization of the low aspect ratio wing's tip vortices, wind gusts the same order of magnitude as the original flight speed. Even in steady freestream, dynamic membrane model computations by Lian [34] and Lian et al. [35], it was shown that the membrane wing experienced high frequency vibrations. NavierStokes simulations and experimental studies report that for membrane type flexible structures under typical MAV Reynolds numbers, the structural response is around $\mathrm{O}(100$ $\mathrm{Hz}$ [1]. These issues, along with stable weight management and flight control problems that intensify with decreasing vehicle sizes, can be potentially attenuated with proper load-redistribution over a membrane wing.

Along with a rigid wing for baseline comparisons, two flexible-wing structures are considered here. First, membrane wings with several chordwise batten structures and a free trailing edge for geometric twist (batten-reinforced wings, BR). Secondly, membrane wings whose interior freely deforms and is sealed along the perimeter to a stiff laminate for aerodynamic twist (perimeter-reinforced wings, PR). Typical flow structures for all three are given in Figure 28., at $15^{\circ}$ angle of attack and $15 \mathrm{~m} / \mathrm{s}$. The two predominate hallmarks of MAV aerodynamics can be seen from the flow over the rigid wing: the low Reynolds number $\left(10^{5}\right)$ causes the laminar boundary layer to separate against the adverse pressure gradient at the root, and the low aspect ratio (1.2) forces a strong wing tip vortex swirling system, and leaves a low pressure cell at the wing tip.

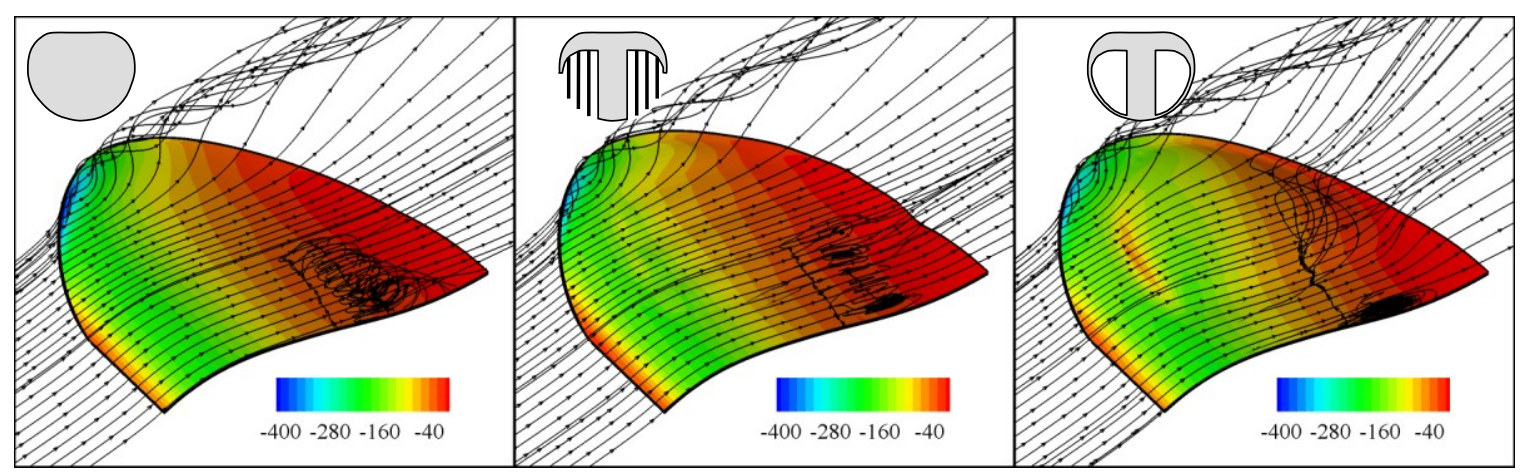

Figure 28. Streamlines and pressure distributions (Pa) over the top wing surface: $\alpha=15^{\circ}$, $\mathrm{U}_{\infty}=15 \mathrm{~m} / \mathrm{s}$.

Flow over the flexible BR wing is characterized by pressure undulations over the surface [36], where the membrane inflation between each batten slightly re-directs the flow. The 
adaptive washout decreases the strength of the adverse pressure gradient, and thus the size of the separation bubble. A large pressure spike develops over the PR wing, at the leading edge of the membrane skin. This is similar to the stagnation point at the leading edge of the wing: flow must decelerate to redirect itself over the tangent discontinuity. The pressure recovery over the wing is shifted aft-ward, and flow separates as it travels down the inflated shape, where it is then entrained into the low-pressure core of the tipvortex. This interaction between the tip vortices and the longitudinal flow separation is known to lead to unsteady vortex destabilization at high angles of attack [37]; no such relationship is obvious over the BR and rigid wings. The low-pressure cells at the wing tips of the two membrane wings are weaker than computed for the rigid wing, presumably due to energy considerations: strain energy in the membrane may remove energy from the lateral swirling system. Furthermore, the inflated membrane shape may act as a barrier to the tip vortex formation.

The lift, drag, and pitching moment coefficients through an $\alpha$-sweep can be seen in Figure 29. The $C_{L}-\alpha$ relationships are mildly nonlinear $\left(20 \%-25 \%\right.$ increase in $C_{L \alpha}$ between $0^{\circ}$ and $15^{\circ}$ ) due to growth of the low pressure cells at the wing tip. Further characteristics of a low aspect ratio are given by the high stall angle, computed at about $21^{\circ}$ (rigid). The aerodynamic twist of the PR wing increases $C_{L \alpha}$ (by as much as $8 \%$ ), making the MAV more susceptible to gusty conditions. $C_{L, m a x}$ is slightly higher as well, subsequently lowering the stall angle to $18^{\circ}$. The adaptive washout of the $\mathrm{BR}$ wing decreases $\mathrm{C}_{\mathrm{L} \alpha}$ (by as much as $15 \%$ over the rigid wing), though the change is negligible at lower angles of attack. This is thought to be a result of two offsetting factors: the adaptive washout at the trailing edge decreases the lift, while the inflation of the membrane towards the leading edge increases the effective camber, and hence the lift.

Comparing the drag polars of Figure 29, it can be seen that both flexible wings incur a drag penalty at small lift values, indicative of the aerodynamically non-optimal shapes assumed by the flexible wings (though the BR wing has less drag at a given angle of attack [32]). As above, the drag difference between the rigid and BR wing is very small, while the PR wing displays a larger penalty. This is presumably due to two factors: a greater percentage of the wing experiences flow separation, and a large portion of the pressure spike at the leading edge is pointed in the axial direction. Pitching moments (measured about the leading edge) have a negative slope with both $\mathrm{C}_{\mathrm{L}}$ and $\alpha$, as necessitated by stability requirements. Nonlinear trends due to low aspect ratio affects are again evident. Both the $\mathrm{BR}$ and the $\mathrm{PR}$ wings have a lower $\partial \mathrm{C}_{\mathrm{m}} / \partial \mathrm{C}_{\mathrm{L}}$ than the rigid wing, though only the PR wing shows a drastic change (by as much as $15 \%$ ). This is a result of the membrane inflation, which shifts the pressure recovery towards the trailing edge, adaptively increasing the strength of the restoring pitching moment with increases in lift/ $\alpha$ [33].

Steeper $\mathrm{C}_{\mathrm{m}}$ slopes indicate larger static margins: stability concerns are a primary target of design improvement from one generation of micro air vehicles to the next. The range of flyable CG locations is generally only a few millimeters long; meeting this requirement represents a strenuous weight management challenge. Furthermore, the PR wing displays a greater range of linear $\mathrm{C}_{\mathrm{m}}$ behavior, possibly due to the fact that the adaptive membrane 
inflation quells the strength of the low-pressure cells, as discussed above. No major differences appear between the L/D characteristics of the three wings for low angles of attack. At moderate angles, the large drag penalty of the PR wing decreases the efficiency, while the BR wing slightly out-performs the rigid wing. At higher angles, both the lift and drag characteristics of the PR wing are superior to the other two, resulting in the best $\mathrm{L} / \mathrm{D}$ ratios.
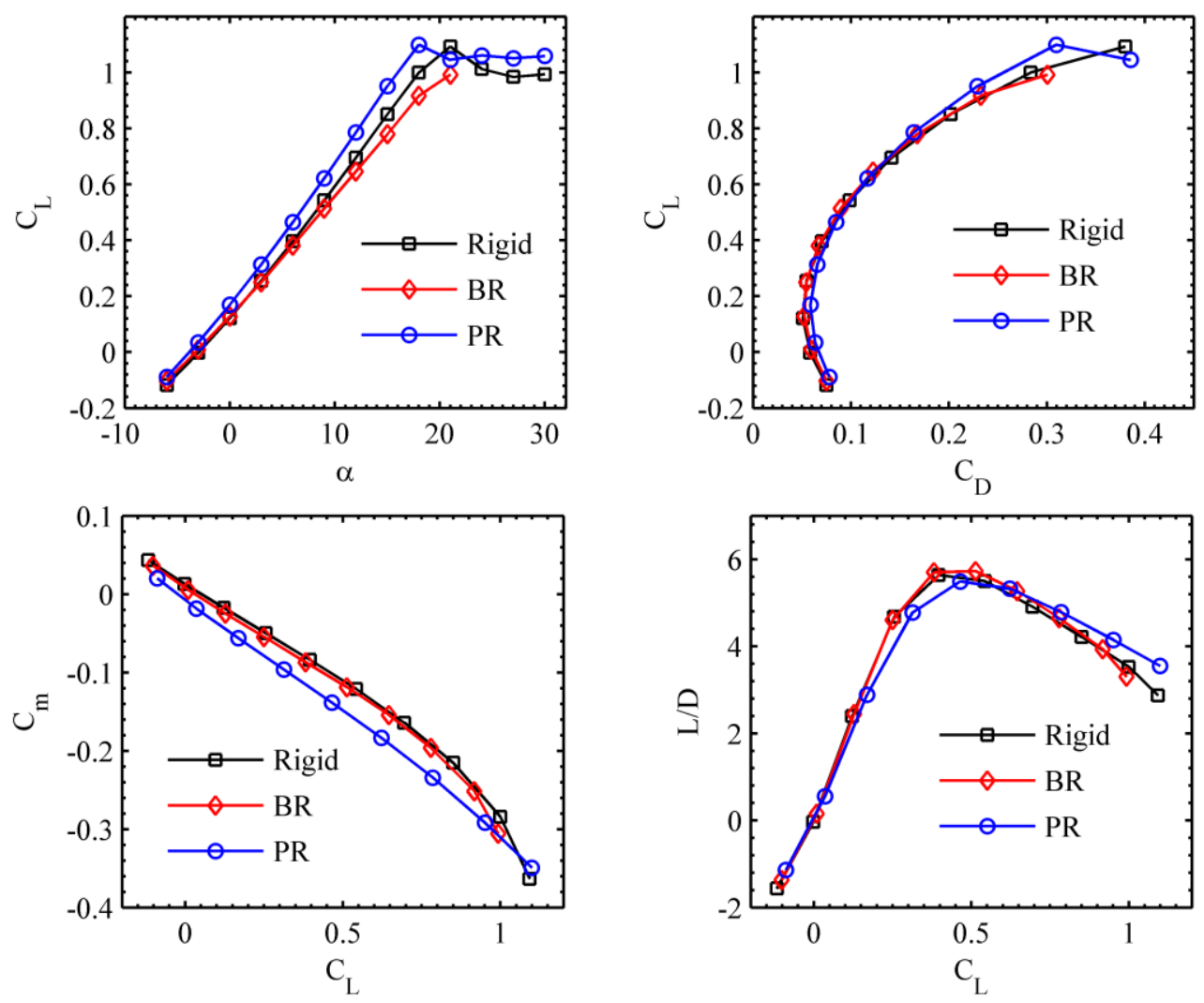

Figure 29. Computed aerodynamic performance: $\alpha=15^{\circ}, \mathrm{U}_{\infty}=15 \mathrm{~m} / \mathrm{s}$.

Aeroelastic tailoring conventionally utilizes unbalanced laminates for bend/twist coupling, but the pre-tension within the membrane skin has an enormous impact on the aerodynamics: for the two-dimensional case, higher pre-tension generally pushes flexible wing performance to that of a rigid wing. For a three-dimensional wing, the response can be considerably more complex, depending on the nature of the membrane reinforcement. Effects of increasing the membrane pre-tension may include: decrease in drag, decrease in $\mathrm{C}_{\mathrm{L} \alpha}$, linearized lift behavior, increase in the zero-lift angle of attack, and more abrupt stalling patterns. Furthermore, aeroelastic instabilities pertaining to shape hysteresis at low angles of attack can be avoided with specific ratios of spanwise-to-chordwise pretensions [38].

Increasing the pre-stress within the membrane skin of a BR wing (Figure 30) generally increases $C_{L \alpha}$, decreases $C_{m \alpha}$, and decreases $L / D$. The system is very sensitive to changes in the pre-stress normal to the battens, and less so to the stress parallel to the battens, due 
to the zero-pre-stress condition at the free edge. Minimizing $\mathrm{C}_{\mathrm{L} \alpha}$ (for optimal gust rejection) is found with no pre-stress in the span-direction, and a mild amount in the chord-direction. The unconstrained trailing edge eliminates the stiffness in this area (allowing for adaptive washout), but retains the stiffness towards the leading edge, removing the inflation seen here (and the corresponding increase in lift). Such a tactic reduces the conflicting sources of aeroelastic lift seen in a BR wing. Maximizing $C_{L \alpha}$ (for effective pull-up maneuvers, for example) is obtained by maximizing $\mathrm{N}_{\mathrm{y}}$ and setting $\mathrm{N}_{\mathrm{x}}$ to zero. Conversely, maximizing $\mathrm{C}_{\mathrm{L} \alpha}$ with a constraint on $\mathrm{L} / \mathrm{D}$ might be obtained by maximizing $\mathrm{N}_{\mathrm{x}}$, and setting $\mathrm{N}_{\mathrm{y}}$ to zero.

Opposite trends are seen for a PR wing. Increasing the pre-stress within the membrane skin generally decreases $\mathrm{C}_{\mathrm{L} \alpha}$, increases $\mathrm{C}_{\mathrm{m} \alpha}$, and increases $\mathrm{L} / \mathrm{D}$. The chord-wise prestress has a negligible effect upon the stability derivatives, though both directions contribute equally to an improvement in $\mathrm{L} / \mathrm{D}$. As such, optimization of either derivative with a constraint on L/D could easily be provided by a design with maximum chord-wise pre-tension and a slack membrane in the span direction. Overall sensitivity of the aerodynamics to the pre-tension in the membrane skin of a BR or a PR wing can be large for the derivatives (up to a $20 \%$ change in the $\mathrm{C}_{\mathrm{m} \alpha}$ of a $\mathrm{BR}$ wing), though less so for the wing efficiency. Variations in $\mathrm{L} / \mathrm{D}$ are never more than $5 \%$.
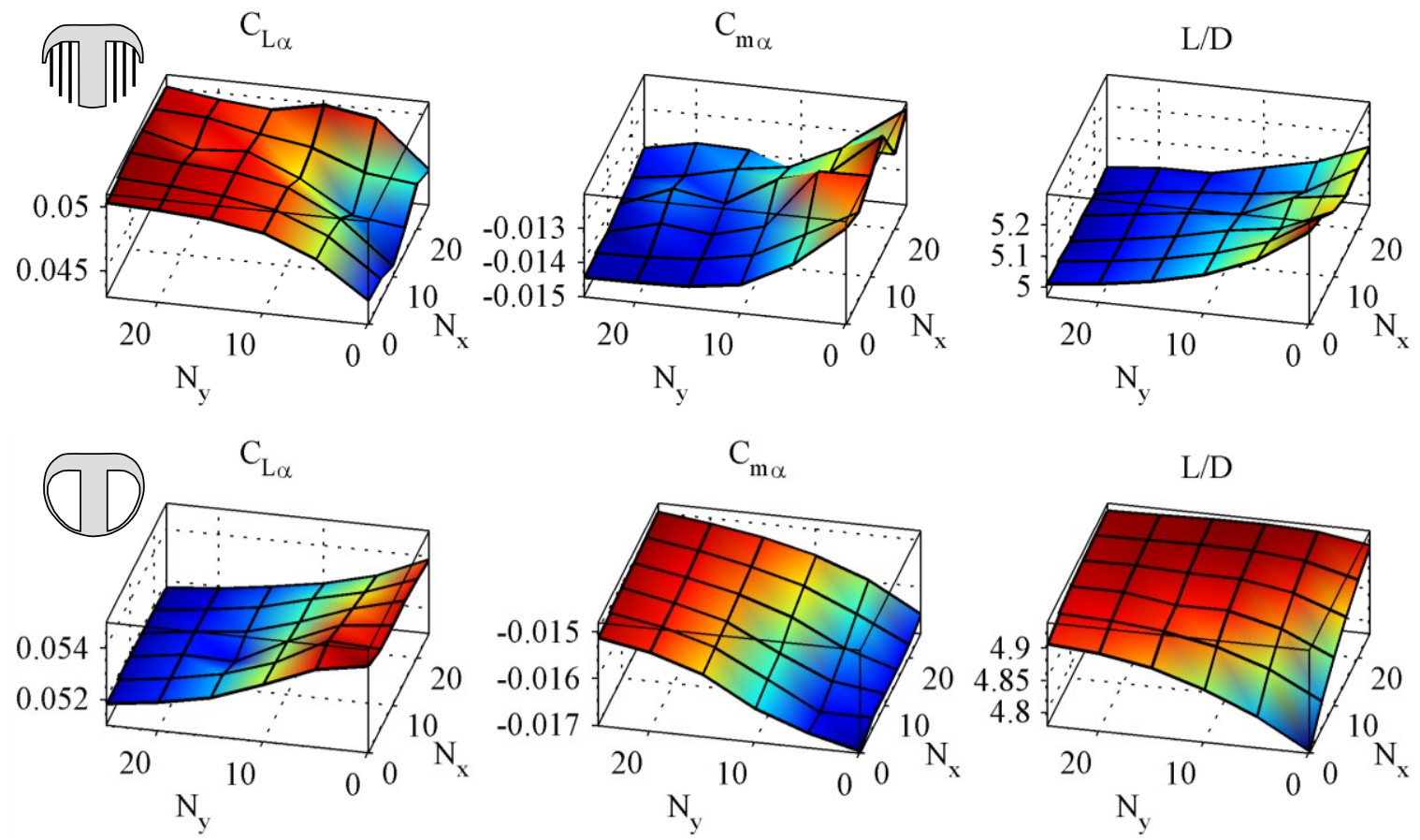

Figure 30. Aeroelastic tailoring of chordwise $\left(\mathrm{N}_{\mathrm{x}}\right)$ and spanwise $\left(\mathrm{N}_{\mathrm{y}}\right)$ membrane prestress resultants $(\mathrm{N} / \mathrm{m})$ : contour represents z-axis values. 


\section{SUMMARY AND CONCLUDING REMARKS}

Based on our recent efforts, the following observations regarding the various aspects of MAV aerodynamics can be summarized.

\section{Leading Edge Vortex}

Three-dimensional Navier-Stokes computations show that LEVs are common at Re of $\mathrm{O}\left(10^{4}\right)$ or lower, which corresponds to the insect flight regime. For hawkmoth which flies at $\mathrm{Re}$ of $\mathrm{O}\left(10^{3}\right.$ to $\left.10^{4}\right)$, LEV along with flapping motion results in enhancement of lift. LEV has a conical shape and breaks down at $75 \%$ of spanwise direction towards wing tip. Also, substantial spanwise pressure gradient promotes delayed stall, whereas spanwise axial flow can stabilize the LEV. For fruit fly in the Re regime of $O(100)$, downwash from the tip vortex and wake vorticity limit the growth of LEV due to effective AoA and prolong the attachment of LEV. The spanwise axial flow is weaker and the spanwise pressure gradient is also less. In translational and rotational motions of a flapping wing the influence of wing rotation is more evident for fruit flies than for hawkmoths. LEV is also stable: LEV is attached during entire downstroke, breaks down during supination. The downwash for hovering is generated from two jets and the vortex tube ring consisting of horseshoe vortex from wing tip, LEV, and TEV.

\section{Aerodynamics and Flapping Kinematics}

Different flow structures are strongly influenced by flapping wing kinematics, stroke amplitude, frequency, Re, and freestream environment. To probe the impact of some of the key kinematics parameters on aerodynamics, a surrogate model is constructed for Re of 100. It is observed that the delayed stall lift peak occurs during surprisingly intense wake interactions and makes the dominant contribution to lift. The peak associated only with wake capture is often not as influential on the lift characteristics as the "valley" between the peaks associated with delayed stall and wake capture. Considering three parameters: plunging amplitude $h_{a}$, angular amplitude $\alpha_{a}$, and phase lag $\phi$, the surrogate model reveals that within the ranges considered, low angular amplitude (i.e. higher angles of attack) and advanced rotation $\left(\phi>90^{\circ}\right)$ generally lead to higher integrated lift values. While the plunging amplitude effects are non-negligible, they are significantly less influential compared to the other two design variables.

\section{Wind Gust and Multiple Time Scales}

For $\mathrm{Re}$ of $\mathrm{O}\left(10^{4}\right)$ or lower, characteristic flapping time scale of insects and birds are much shorter than that of typical gust. Flapping wing can alleviate gust effect to provide better platform for the vehicle.

\section{Fluid-Structure Interactions and Membrane Wing}

Insect, bat and bird wings are characterized by anisotropic structural properties. For insects, the spanwise bending stiffness is of 1 to 2 orders of magnitude larger than chordwise one. Complex interplay of geometrically nonlinear effects and anisotropy is critical for the success of future MAV designs. Forward flight with flexible plunging airfoil has phase difference relative to pitching. This will result in equivalent pitching angle affecting the aerodynamic coefficients. Membrane wing behaves like passive shape 
optimization through geometric or aerodynamic twist. Geometrically, streamlining alleviates maneuver/gust loads and delays the onset of stall by nose-down rotation of each wing section. On the other hand, aerodynamic twist tend to increase the maximum $C_{L}$, longitudinal static stability, and drag by rotation of zero-lift AoA via load-induced membrane inflation. Comparison of rigid wing, batten-reinforced wings, and perimeterreinforced wings show that both membrane wings incur drag penalty at small lift values.

Observing the fast growing papers and designs available in the open domain, it seems clear that strong attempts are being made in the research and development community to lay foundation for the advancement of MAVs. While much progress has been made, more advancement is needed before we can develop robust and agile MAV technologies. In particular, the following computational and experimental endeavors are needed and should be pursued.

- There is a fundamental need for improving our understanding of the fluid physics of biology-inspired mechanisms that simultaneously provide lift and thrust, enable hover, and provide high flight control authority, while minimizing power consumption. First principles-based computational modeling and analysis capabilities are essential in support of the investigation of issues related to fluid-structure interactions, laminar-turbulent transition, unsteady freestream (wind gust), and time dependent aerodynamics.

- We need to conduct further exploration of flexible, light-weight, multifunctional materials and structures for large displacement and suitability for actuators and sensors.

- Bio-inspired mechanisms need to be developed for flapping wing. These mechanisms will include both joints and distributed actuation to enable flapping and morphing. Most importantly, the motion produced by these mechanisms should be experimented based on what we can learn from biological systems performing flapping in gusty conditions.

- The fluid flow associated with these mechanisms need be detailed using a rigorous set of experiments. These measurements will include simultaneous flow field and structural deformation measurements in order to better understand the causal relationships between them.

- Vision-based sensing techniques need to be developed to estimate the aeroelastic states of the vehicle. Estimates of both rigid-body and deformation states can be extracted by noting frequency-varying properties of optical flow. This synthesis will address stability and performance metrics for the nonlinear dynamics and time-varying properties.

- Gust-tolerant biology-inspired flight control methodologies incorporating novel sensors and wing structural property tailoring need to be advanced.

Acknowledgment: The present work is supported by a Multidisciplinary University Research Initiative (MURI) project sponsored by AFOSR.

\section{$\underline{\text { References }}$}

1. Shyy, W., Lian, Y., Tang, J., Viieru, D. and Liu, H. Aerodynamics of Low Reynolds Number Flyers, Cambridge University Press, New York, 2008.

2. Wakeling, J. M, and Ellington, C. P. "Dragonfly flight. I. Gliding flight and steady-state aerodynamic forces," J Exp Biol. , Vol. 200, 1997, pp. 543-556. 
3. http://turbulence.kmip.net/

4. Platzer, M. and Jones, K.D., "Flapping Wing Aerodynamics - Progress and Challenges," $44^{\text {th }}$ AIAA Aerospace Sciences Meeting and Exhibit, Paper No. 2006-500, 2006.

5. Liu, H. and Kawachi, K., "A numerical study of insect flight," J. Comp. Physics., Vol. 146, 1998, pp. 124-156.

6. Ramamurti, R. and Sandberg, W.C., "A Three-Dimensional Computational Study of the Aerodynamic Mechanisms of Insect Flight," J. Exp. Biol., Vol. 205, 2002, pp. 1507-1518.

7. Lian, Y., and Shyy, W., "Aerodynamics of Low Reynolds Number Plunging Airfoil under Gusty Environment," AIAA Paper 2007-71, Reno, NV, 2007.

8. Ellington, C.P., Van den Berg, C., Willmott, A.P. and Thomas, A.L.R. "Leadingedge Vortices in Insect Flight," Nature, Vol. 384, 1996, pp. 626-630.

9. Van den Berg, C. and Ellington, C.P. "The Three-dimensional Leading-edge Vortex of a 'Hovering' Model Hawkmoth," Philosophical Transactions of the Royal Society of London. Series B, Vol. 352, 1997, pp. 329-340.

10. $\quad$ Srygley, R.B. and Thomas, A.L.R. " Unconventional Lift-generating Mechanisms in Free-flying Butterflies," Nature, Vol. 420, 2002, pp. 660-664.

11. Thomas, A.L.R., Taylor, G.K., Srygley, R.B., Nudds, L.R. and Bomphrey, R.J. "Dragonfly Flight: Free-flight and Tethered Flow Visualizations Reveal a Diverse Array of Unsteady Lift-generating Mechanisms, Controlled Primarily Via Angle of Attack," Journal of Experimental Biology, Vol. 207, 2004, pp. 4299-4323.

12. Birch, J.M. and Dickinson, M.H. "Spanwise Flow and the Attachment of the Leading-edge Vortex on Insect Wings," Nature, Vol. 412, 2001, pp. 729-733.

13. Zbikowski, R. "On Aerodynamic Modelling of an Insect-like Flapping Wing in Hover for Micro Air Vehicles," Philosophical Transactions of the Royal Society of London. Series A, Vol. 360, 2002, pp. 273-290.

14. McCroskey, W.J., McAlister, K.W., Carr, L.W. and Pucci, S.L. "An Experimental Study of Dynamic Stall on Advanced Airfoil Section," NASA TM-84245, 1982.

15. De Vries, O. "On the Theory of the Horizontal-Axis Wind Turbine," Annual Review of Fluid Mechanics, Vol. 15, 1983, pp. 77-96.

16. McCroskey, W.J., Carr, L.W. \& McAlister, K.W. "Dynamic Stall Experiments on Oscillating Airfoils," AIAA Journal, Vol. 14, 1976, pp. 57-63.

17. Shyy, W. and Liu, H., "Flapping Wings and Aerodynamic Lift: The Role of Leading-Edge Vortices," AIAA Journal, Vol. 45, (2007), pp. 2817-2819.

18. Dickinson, M. H., and Götz, K. G., "Unsteady Aerodynamic Performance of Model Wings at Low Reynolds Numbers," Journal of Experimental Biology, Vol. 174, Jan. 1993, pp. 45-64.

19. Ellington, C. P., Berg, C. van den, Willmott, A. P., and Thomas, A. L. R., "Leading-edge Vortices in Insect Flight," Nature, Vol. 384, Dec. 1996, pp. 626-630.

20. Lehmann, F-O., "The Mechanisms of Lift Enhancement in Insect Flight," Naturwissenschaften, Vol. 91, 2004, pp. 101-122.

21. Viieru, D., Tang, J., Lian, Y., Liu, H., and Shyy, W., Flapping and Flexible Wing Aerodynamics of Low Reynolds Number Flight Vehicles, 44th AIAA Aerospace Sciences Meeting and Exhibit, AIAA paper 2006-0503. 
22. Visbal, M. R., Gordnier, R. E., "Numerical Simulation of the Interaction of a Transitional Boundary Layer with a 2-D Flexible Panel in the Subsonic Regime," Journal of Fluids andStructures, Vol. 19, No.7, 2004, pp. 881-903.

23. Yuan, W., Khalid, M., Windte, J., Scholz, U., and Radespiel, R., "An Investigation of Low-Reynolds-Number Flows Past Airfoils," AIAA Paper 2005-4607, 23rd AIAA Applied Aerodynamics Conference, Toronto, June 6-9, 2005.

24. Lian, Y. and Shyy, W., "Laminar-Turbulent Transition of a Low Reynolds Number Rigid or Flexible Airfoil," AIAA 36th Fluid Dynamics Conference and Exhibit, 2006, Paper No. 2006-3051.

25. Radespiel, R., Windte, J., and Scholz, U.,"Numerical and Experimental Flow Analysis of Moving Airfoil with Laminar Separation Bubbles, " AIAA Paper 2006-0501.

26. Ol, M., McAuliffe, B. R., Hanff, E. S., Scholz, U., Kaehler, Ch., "Comparison of Laminar Separation Bubble Measurements on a Low Reynolds Number Airfoil in Three Facilities", AIAA 2005-5149, 2005.

27. Shyy, W., Berg, M. and Ljungqvist, D., "Flapping and Flexible Wings for Biological and Micro Air Vehicles," Progress in Aerospace Sciences, Vol. 35, 1999, pp. 155-205.

28. Shyy, W., Ifju, P.G. and Viieru, D., "Membrane Wing-Based Micro Air Vehicles," Applied Mechanics Reviews, Vol. 58, 2005, pp.283-301.

29. Combes, S. A., and Daniel, T. L., "Flexural Stiffness in Insect Wings I. Scaling and the Influence of Wing Venation," Journal of Experimental Biology, Vol. 206, 2003, pp. 2979-2987.

30. Heathcote, S., and Gursul, I., "Flexible Flapping Airfoil Propulsion at Low Reynolds Numbers," AIAA Paper 2005-1405, Jan. 2005.

31. Tang, J., Viieru, D. and Shyy, W., "A Study of Aerodynamics of Low Reynolds Number Flexible Airfoils," AIAA Paper No. 2007-4212, 37th AIAA Fluid Dynamics Conference and Exhibit, 2007.

32. Argentina, M., Mahadevan, L., "Fluid-Flow-Induced Flutter of a Flag," Proceedings of the National Academy of Science: Applied Mathematics, Vol. 102, No. 6, pp.1829-1834, 2005.

33. Stanford, B., Sytsma, M., Albertani, R., Viieru, D., Shyy, W., Ifju, P., "Static Aeroelastic Model Validation of Membrane Micro Air Vehicle Wings," AIAA Journal, Vol. 45, No. 12, pp. 2828-2837, 2007.

34. Lian, Y., Membrane and Adaptively-Shaped Wings for Micro Air Vehicles, Ph.D thesis, University of Florida, Gainesville, 2003.

35. Lian, Y., Shyy, W., Viieru, D. and Zhang, B., "Membrane Wing Aerodynamics for Micro Air Vehicles," Progress in Aerospace Sciences, Vol. 39, (2003), pp. 425-465.

36. Hepperle, M., "Aerodynamics of Spar and Rib Structures," MH AeroTools Online Database, http://www.mh-aerotools.de/airfoils/ribs.htm, March 2007.

37. Tang, J., Zhu, K., "Numerical and Experimental Study of Flow Structure of LowAspect Ratio Wing," Journal of Aircraft, Vol. 41, No. 5, pp. 1196-1201, 2004.

38. Ormiston, R., "Theoretical and Experimental Aerodynamics of the Sail Wing," Journal of Aircraft, Vol. 8, No. 2, pp. 77-84, 1971. 